\title{
Dissipative hydrodynamics with higher-form symmetry
}

\author{
Jay Armas, ${ }^{a, b}$ Jakob Gath, ${ }^{c}$ Akash Jain ${ }^{d}$ and Andreas Vigand Pedersen ${ }^{e}$ \\ ${ }^{a}$ Institute for Theoretical Physics, University of Amsterdam, \\ 1090 GL Amsterdam, The Netherlands \\ ${ }^{b}$ Dutch Institute for Emergent Phenomena, University of Amsterdam, \\ 1090 GL Amsterdam, The Netherlands \\ ${ }^{c}$ Department of Physics, Technical University of Denmark, \\ DK-2800 Kgs. Lyngby, Denmark \\ ${ }^{d}$ Centre for Particle Theory $\&$ Department of Mathematical Sciences, Durham University, \\ South Road, Durham DH1 3LE, United Kingdom \\ e Seaborg Technologies, \\ Bredgade 36B, 1260 Copenhagen, Denmark \\ E-mail: j.armas@uva.nl, jagath@fysik.dtu.dk, akash.jain@durham.ac.uk, \\ andreas@seaborg.co
}

ABSTRACT: A theory of parity-invariant dissipative fluids with $q$-form symmetry is formulated to first order in a derivative expansion. The fluid is anisotropic with symmetry $\mathrm{SO}(D-1-q) \times \mathrm{SO}(q)$ and carries dissolved $q$-dimensional charged objects that couple to a $(q+1)$-form background gauge field. The case $q=1$ for which the fluid carries string charge is related to magnetohydrodynamics in $D=4$ spacetime dimensions. We identify $q+7$ parity-even independent transport coefficients at first order in derivatives for $q>1$. In particular, compared to the $q=1$ case under the assumption of parity and charge conjugation invariance, fluids with $q>1$ are characterised by $q$ extra transport coefficients with the physical interpretation of shear viscosity in the $\mathrm{SO}(q)$ sector and current resistivities. We discuss certain issues related to the existence of a hydrostatic sector for fluids with higher-form symmetry for any $q \geq 1$. We extend these results in order to include an interface separating different fluid phases and study the dispersion relation of capillary waves finding clear signatures of anisotropy. The formalism developed here can be easily adapted to study hydrodynamics with multiple higher-form symmetries.

Keywords: Effective Field Theories, Holography and condensed matter physics (AdS/CMT), Holography and quark-gluon plasmas

ARXIV EPRINT: 1803.00991 


\section{Contents}

1 Introduction 1

1.1 Summary of the results and organisation of the material 3

2 Ideal order fluids with $q$-form symmetry 4

2.1 Fluid stress tensor and current 5

$\begin{array}{lll}2.2 & \text { Thermodynamics } & 6\end{array}$

$\begin{array}{ll}2.3 \text { Conservation equations } & 6\end{array}$

3 Dissipative fluids with $q$-form symmetry $\quad 8$

3.1 Dissipative corrections and choices of fluid frames 9

$\begin{array}{lll}3.2 & \text { Entropy current constraints } & 11\end{array}$

$\begin{array}{lll}3.3 & \text { Kubo formulae } & 14\end{array}$

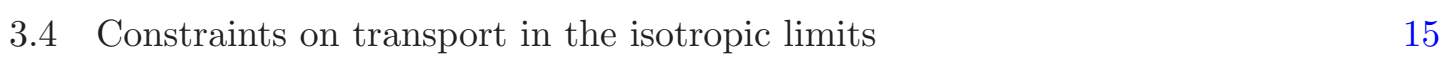

4 Equilibrium partition function $\quad 16$

$\begin{array}{ll}4.1 \text { The partition function } & 16\end{array}$

$\begin{array}{ll}\text { 4.2 Additional constraints from the second law of thermodynamics } & 18\end{array}$

5 Surface dynamics of fluids with $q$-form symmetry 20

5.1 Conservation equations and the second law of thermodynamics 21

5.2 Equilibrium partition function and entropy current analysis 22

$\begin{array}{lll}5.3 & \text { Surface conservation equations } & 23\end{array}$

$\begin{array}{lll}5.4 & \text { Surface waves } & 23\end{array}$

$\begin{array}{llr}6 & \text { Discussion } & 27\end{array}$

$\begin{array}{ll}\text { A Currents in another fluid frame } & 29\end{array}$

\section{Introduction}

The microscopic description of charged quantum matter is usually intractable when the number of its fundamental objects is very large. Generically, however, such microscopic descriptions admit a hydrodynamic limit in which the low-energy collective behaviour of matter is captured by a few emergent degrees of freedom, such as temperature, chemical potential and velocity fields. In this limiting regime, rapidly varying quantities compared to the mean-free path of the fundamental objects are integrated out while the dynamics of the slowly varying quantities is governed by the conservation laws of the microscopic system. These conservation laws are a direct manifestation of the underlying symmetries of the system. 
Despite hydrodynamics being a well established research subject, there has been a substantial progress in its structural foundations in recent years. This includes an off-shell formulation of hydrodynamics and the development of classification schemes of its transport properties [1-3]; the construction of effective field theories and the formulation of action principles for dissipative fluid dynamics [4-9]; the establishment of a framework for describing interfaces between different fluid phases [10-12]; a new formalism for studying non-relativistic fluids [13-16]; and the development of hydrodynamic theories with generalised global 1-form symmetries and their connections to magnetohydrodynamics [17-20] as well as their role in the understanding of effective theories with translational symmetry breaking and states with dynamical defects [21].

This paper introduces a framework for building effective hydrodynamic theories of dissipative fluids with $q$-form symmetries, generalising previous work for $q=0,1$. These effective theories correspond to the hydrodynamic limit of microscopic descriptions whose underlying fundamental charged objects are $q$-dimensional (i.e. $q$-dimensional branes). These $q$-dimensional objects couple to a background gauge field $A_{q+1}$. In the language of [22], these fluids describe microscopic systems with a generalised $q$-form global symmetry. Associated with the $q$-form symmetry is a $(q+1)$-form current $J$ whose integral over a $(D-q-1)$ dimensional hypersurface $\mathcal{M}_{\Gamma}$ yields a conserved dipole charge

$$
Q_{\mathcal{M}_{\Gamma}}=\int_{\mathcal{M}_{\Gamma}} \star J
$$

where the operator $\star$ is the Hodge dual operator in $D$-dimensional spacetime. This dipole charge counts the number of $q$-dimensional objects that cross the $(D-q-1)$-dimensional hypersurface $\mathcal{M}_{\Gamma} \cdot{ }^{1}$ The hydrodynamic theories constructed here capture the collective excitations of these charged $q$-dimensional objects around a state of thermal equilibrium.

This work has been highly motivated by the structure of long-wavelength perturbations of black branes in supergravity, where dissipative fluids with higher-form symmetries are naturally realised [23-27]. As the fundamental fields in supergravity include several higherform gauge fields, generic black brane bound states can carry multiple higher-form (dipole) charges. For instance, the D3-F1 bound state in type IIB string theory carries two 2form currents and one 4-form current [27]. Within this context, the fluid stress tensor and charge currents appear as the effective currents sourcing the gravitational and electric fields far away from the black brane horizon [27], while their conservation laws are realised as constraint equations when solving Einstein equations in a long-wavelength expansion.

Tackling the general problem of establishing a hydrodynamic theory of dissipative fluids carrying multiple higher-form charges is certainly of interest and in this work we take the first step towards this goal by constructing the effective hydrodynamic theory of fluids with one single $q$-form symmetry. These fluids are anisotropic with $\operatorname{SO}(D-q-1) \times \operatorname{SO}(q)$ symmetry and the source of this anisotropy is the existence of a higher-form charge current. Gravitational duals to these fluids are encountered in black brane geometries in gravity

\footnotetext{
${ }^{1}$ In the case $q=1$ in $D=4$ and in the context of magnetohydrodynamics where $J_{\mu \nu}=\star F_{\mu \nu}$, the fundamental objects are strings and $Q_{\mathcal{M}_{\Gamma}}$ counts the number of magnetic field lines crossing a codimension-2 hypersurface. See [18] for a detailed discussion.
} 
theories with a metric $g_{\mu \nu}$, one single $(q+1)$-form gauge field $A_{q+1}$ and possibly a dilaton field. Several examples of such gravitational duals with arbitrary $q$ were found in [24].

In this work, we formulate the theory of dissipative fluids with $q$-form symmetry to first order in a long-wavelength expansion (to first order in derivatives of the fluid fields) focusing on the parity-even sector of the theory. ${ }^{2}$ We also study equilibrium configurations and highlight some of the technical complications that arise while describing the hydrostatic sector of fluids with higher-form symmetries. In addition, we generalise our results to include the presence of interfaces separating different fluid phases and study specific cases of surface waves, finding signatures of anisotropy in their dispersion relation.

\subsection{Summary of the results and organisation of the material}

In section 2, the ideal order dynamics of fluids with $q$-form symmetry living in a background spacetime with metric $g_{\mu \nu}$ and a $(q+1)$-form gauge field $A_{q+1}$ is introduced. These fluids are anisotropic with $\mathrm{SO}(D-q-1) \times \mathrm{SO}(q)$ symmetry and carry a dipole charge density $Q$. They are characterised by a stress tensor, charge $(q+1)$-form current and an entropy current. The existence of this $(q+1)$-form current is responsible for the microscopic anisotropic properties of the fluid. When the charge $Q$ vanishes, the current vanishes as well and the usual uncharged isotropic fluid is recovered. We establish their thermodynamic properties and conservation laws. As far as we are aware, this is the first time that the ideal order dynamics of these fluids is formalised in the literature.

In section 3 we formulate the dissipative sector of the theory up to first order in derivatives. We focus on the parity-even sector for $q \geq 0$ while for $q=1$ we in addition require charge conjugation invariance. First, we describe how frame transformations act on the stress tensor and currents and comment on different choices of frames. Picking a higher-form analogue of the Landau frame, we proceed and require the divergence of the entropy current to be positive semi-definite. This leads to the existence of $q+8$ transport coefficients for $q>1$ and 8 for $q=1$, all of which are dissipative, thereby contributing to entropy production. Once Kubo formulae are obtained, we note that Onsager's relation for mixed correlation functions sets a constraint among these transport coefficients, thereby leading to $q+7$ independent transport coefficients at first order in derivatives for $q>1$ and 7 for $q=1$. Compared to the $q=1$ case studied in [18, 19], for $q>1$ there is one additional transport coefficient with the physical interpretation of shear viscosity in the $\mathrm{SO}(q)$ sector and $q-1$ extra current resistivities. At the end of this section, we study the constraints on these transport coefficients in isotropic limits. We observe that some of these constraints are satisfied by gravitational duals even away from the isotropic limits.

Section 4 contains a detailed analysis of equilibrium configurations in theories with a $q$-form symmetry. We begin by noting that the equilibrium partition function presented in [18] for $q=1$ does not describe the hydrostatic sector of the theory completely. As such, the hydrostatic solution as defined in [18], which assumes hydrostatic backgrounds to admit not just a timelike isometry but also $q$ spacelike isometries, causes production of

\footnotetext{
${ }^{2}$ The $q=1$ case deserves considerably more attention and will be the focus of a later publication [28]. In this paper we further restrict the $q=1$ case by requiring charge conjugation invariance in addition to parity invariance. In this case, our analysis for $q=1$ is the same as that inpro [18].
} 
entropy, which is incompatible with equilibrium. We show that to avoid this, an additional constraint must be imposed on the hydrostatic backgrounds. Furthermore, [18] assumed that spacelike and timelike isometries admitted by the hydrostatic backgrounds must have vanishing mutual inner products, which further restricts the class of backgrounds on which equilibrium can be realised. We explicitly derive the most general partition function where these inner products are not assumed to be zero and show that the resulting solution is a $q$-form generalisation of the $q=1$ solution presented in [24]. We comment on other issues regarding the hydrostatic sector of these theories, wherein the requirement of an equilibrium partition function seems to impose more constraints than requiring the second law of thermodynamics alone to hold. This is in striking contrast with $q=0$ hydrodynamics, where the second law guarantees the existence of an equilibrium partition function at all orders in the derivative expansion [29, 30].

In section 5 , following $[10,12]$, we generalise our results in order to include the presence of an interface/surface separating two different fluid phases. Similar to section 4 , we write down a partition function for fluids with $q$-form symmetry in the presence of the interface. We then analyse the divergence of the surface entropy current and obtain the surface thermodynamics as well as a constraint on the normal component of the bulk fluid velocity. We observe that this matches partition function expectations. Having established a notion of equilibrium in this setting, we obtain the dispersion relation for capillary waves and ripples on the interface, finding clear signals of anisotropy.

Finally, in section 6 we comment on some open issues and future research directions. We also provide appendix A with some of our results written in another fluid frame in order to ease comparison with earlier literature.

\section{Ideal order fluids with $q$-form symmetry}

In this section we introduce the ideal order currents and conservation equations for the propagation of an anisotropic fluid with $q$-form symmetry carrying $q$-brane charge in a $D$-dimensional background geometry $\left(\mathcal{M}, g_{\mu \nu}, A_{q+1}\right)$ with $p$-spatial directions so that $D=(p+1)$ with $p \geq q$. The manifold $\mathcal{M}$ is endowed with the Levi-Civita connection $\nabla_{\mu}$ built out from the background metric $g_{\mu \nu}$ with coordinates $x^{\mu}$. These fluids are characterised by a $(q+1)$-form current $J$ that couples to the background gauge field $A_{q+1}$. In general, introducing a conserved higher-form current breaks the $\mathrm{SO}(p)$ symmetry enjoyed by the ordinary relativistic "point charged" (or neutral) fluid to a $\mathrm{SO}(p-q) \times \mathrm{SO}(q)$ symmetry. ${ }^{3}$ As usual, at each point of $\mathcal{M}$ there exists a rest-frame in which the fluid is static. At ideal order, this frame is unambiguously defined and is characterised by a timelike vector $u^{\mu}$ (the fluid velocity) normalised such that $u^{\mu} u_{\mu}=-1$. For later use, we introduce the projector transverse to the fluid velocity $\Delta^{\mu \nu}=g^{\mu \nu}+u^{\mu} u^{\nu}$. The local thermodynamic fields of the fluid are then unambiguously defined as their local values in the rest-frame. We now proceed to write now the ideal order hydrodynamics describing this system.

\footnotetext{
${ }^{3}$ If multiple $q_{i}$-form conserved currents with $i=1, \ldots, \ell$ are introduced, the fluid is expected to have the symmetry $\mathrm{SO}\left(p-\sum_{i=1}^{\ell} q_{i}\right) \times \mathrm{SO}\left(q_{i}\right) \times \cdots \times \mathrm{SO}\left(q_{\ell}\right)$.
} 


\subsection{Fluid stress tensor and current}

At the ideal order level, the $(q+1)$-form current takes the following form

$$
J_{\text {ideal }}=Q \operatorname{Vol}_{(q+1)} .
$$

Here $Q$ denotes the quasi-local dipole charge and $\operatorname{Vol}_{(q+1)}$ is a $(q+1)$-dimensional volume form. We can decompose it as $\operatorname{Vol}_{(q+1)} \equiv u \wedge \operatorname{Vol}_{q}$, where $\operatorname{Vol}_{q}=-\imath_{u} \operatorname{Vol}_{(q+1)}$ (here $\imath$ denotes the interior product) is the local volume form on the space spanned by the $q$ (linearly independent) spatial directions $v^{i}$ of the current in the rest-frame, i.e.

$$
\operatorname{Vol}_{q}=\sqrt{\operatorname{det}\left(v_{i} \cdot v_{j}\right)} v^{1} \wedge \ldots \wedge v^{q}, \quad v_{\mu}^{i} u^{\mu}=0 .
$$

Here we have used the inverse of the matrix $\left(v^{i} \cdot v^{j}\right)$ defined as $g^{\mu \nu} v_{\mu}^{i} v_{\nu}^{j}$, which is the induced metric on the $q$-manifold. We will justify this picture and notation below. Clearly, $\mathrm{Vol}_{q}$ and $u^{\mu}$ are both $\mathrm{SO}(q)$ invariant structures. Moreover, $\mathrm{Vol}_{(q+1)}$ is a $\mathrm{SO}(1, q)$ Lorentz invariant under boosts along the $q$-directions $v^{i}$ with $i=1, \ldots, q$. This invariance must be reflected in the hydrodynamic theory once dissipation is introduced.

In general, the current induces stresses along the $q$-spatial directions of the current. We thus introduce a $\mathrm{SO}(q)$ invariant projector $\Pi_{\mu \nu}$ along these directions. In particular, this projector satisfies the relations

$$
\Pi_{\rho}^{\mu} \Pi_{\nu}^{\rho}=\Pi_{\nu}^{\mu}, \quad \Pi_{\rho}^{\mu} u^{\rho}=0, \quad \operatorname{Tr} \Pi \equiv g^{\mu \nu} \Pi_{\mu \nu}=q .
$$

Without loss of generality, one may choose the $q$ one-forms $v^{i}$ to be orthonormal, leading to the projector ${ }^{4}$

$$
\Pi_{\mu \nu}=\delta_{i j} v_{\mu}^{i} v_{\nu}^{j}, \quad g^{\mu \nu} v_{\mu}^{i} v_{\nu}^{j}=\delta^{i j},
$$

thereby justifying the form of (2.2). Given this projector, we introduce the ideal order fluid stress tensor as

$$
T_{\text {ideal }}^{\mu \nu}=E u^{\mu} u^{\nu}+P_{q} \Pi^{\mu \nu}+P \Gamma^{\mu \nu},
$$

where $\Gamma^{\mu \nu} \equiv \Delta^{\mu \nu}-\Pi^{\mu \nu}$ is the $\mathrm{SO}(p-q)$ invariant projector orthogonal to the current. This projector satisfies $\Gamma^{\mu \rho} \Pi_{\rho \nu}=\Gamma^{\mu \rho} u_{\rho}=0$ and $\operatorname{Tr} \Gamma=(p-q)$. We also find it convenient to introduce the projector onto all timelike and spacelike directions of the charge current, $\Xi^{\mu \nu}=-u^{\mu} u^{\nu}+\Pi^{\mu \nu}$. The stress tensor (2.5) is the most general stress tensor compatible with the given symmetries. In $(2.5)$ we have introduced the energy density $E$ such that $u_{\nu} T_{\text {ideal }}^{\mu \nu}=-E u^{\mu}$ while $P_{q}$ and $P$ denote the pressure along and orthogonal to the current, respectively.

The stress tensor also satisfies the orthogonality condition $\Pi_{\mu \rho} \Gamma_{\nu \sigma} T_{\text {ideal }}^{\mu \nu}=0$, which will play an important role in the choice of fluid frame when discussing dissipative effects. Note that, in particular, when $q=0$ (equivalently $\Pi_{\mu \nu}=0$ ) or when $q=p$ (equivalently $\left.\Gamma_{\mu \nu}=0\right)$ we recover the complete $\mathrm{SO}(p)$ isotropy as required..$^{5}$ Isotropy is also recovered

\footnotetext{
${ }^{4}$ Note that $\mathrm{Vol}_{q}$ is invariant under a local GL $(q)$ transformation: $v_{\mu}^{i} \rightarrow R_{j}^{i} v_{\mu}^{j}$, where $R_{j}^{i}$ is an arbitrary $q \times q$ non-singular matrix. By choosing to work in an orthonormal basis for the vectors $v_{\mu}^{i}$, we have reduced this $\mathrm{GL}(q)$ symmetry down to a residual $\mathrm{SO}(q)$.

${ }^{5}$ The dynamics of $p=q$ fluids has been extensively analysed in $[23,26]$.
} 
in the uncharged (or neutral) limit $(Q=0)$ since the source of anisotropy is only due to the existence of the current (2.1). In turn, this implies that $\left.\left(P-P_{q}\right)\right|_{Q=0}=0$. Requiring consistency with the existence of an uncharged isotropic limit will impose non-trivial constraints among transport coefficients in the dissipative sector of the theory in that limit.

\section{$2.2 \quad$ Thermodynamics}

The local thermodynamics of the $q$-charged fluid is analogous to the local thermodynamics of the $q=1$ fluid first considered in [24]. In particular, the fluid energy density satisfies the first law of thermodynamics

$$
\mathrm{d} E=T \mathrm{~d} S+\mu \mathrm{d} Q
$$

with $T, S$ and $\mu$ denoting the temperature, entropy density and chemical potential dual to $Q$, respectively. ${ }^{6}$ We assume that the fluid carries an entropy current of the form

$$
S_{\text {ideal }}^{\mu}=S u^{\mu},
$$

which we require to obey a local form of the second law of thermodynamics $\nabla_{\mu} S_{\text {ideal }}^{\mu} \geq 0$.

In addition, the difference between the pressure orthogonal and along the current is given by the energy density of the dissolved $q$-branes carried by the fluid, i.e.

$$
P-P_{q}=\mu Q
$$

The local thermodynamics of the fluid with $q$-brane charge also satisfy the Gibbs-Duhem relations

$$
E+P=T S+\mu Q, \quad \mathrm{~d} P=S \mathrm{~d} T+Q \mathrm{~d} \mu, \quad \mathrm{d} P_{q}=S \mathrm{~d} T-\mu \mathrm{d} Q .
$$

One may easily see that relation (2.8) is the result of integrating the last two relations in (2.9). Furthermore, it is clear that in the uncharged limit $(Q=0)$ one obtains the condition $\left.\left(P-P_{q}\right)\right|_{Q=0}=0$.

\subsection{Conservation equations}

The stress tensor and current obey the usual conservation laws

$$
\nabla_{\mu} T^{\mu \nu}=F^{\nu}, \quad \mathrm{d} \star J=0,
$$

where $F^{\nu} \equiv \frac{1}{(q+1) !} F^{\nu \mu_{1} \ldots \mu_{q+1}} J_{\mu_{1} \ldots \mu_{q+1}}$ is a Lorentz force acting on the fluid and where the $(q+2)$-form $F$ is the field strength $F=\mathrm{d} A_{q+1}$. Here $\star$ is the Hodge dual on $\mathcal{M}$. We first consider the charge conservation equation. Taking the wedge product of $\mathrm{d} \star J=0$ with $J$ itself we obtain

$$
\star J \wedge \star(\mathrm{d} \star J)=\star \mathfrak{f}
$$

\footnotetext{
${ }^{6}$ These thermodynamic properties are motivated from black brane geometries [23, 24, 27] but we also show in section 4 that they naturally follow from partition function considerations.
} 
where at ideal order, $\mathfrak{f}$ is the one-form

$$
\mathfrak{f}_{\mu}=(-1)^{q} Q \Xi_{\mu \lambda} \nabla_{\nu}\left(Q \Xi^{\nu \lambda}\right)
$$

By projecting out this equation along the $u_{\mu}$ and $\Pi_{\mu \nu}$ directions, the vanishing of the one-form $\mathfrak{f}$ then implies two continuity equations for the charge $Q$, namely

$$
u^{\mu} \nabla_{\mu} Q=-Q \vartheta_{(p-q)}, \quad \Pi_{\mu}{ }^{\nu} \nabla_{\nu} Q=Q \Pi_{\mu}^{\nu}\left(\mathfrak{a}_{\nu}-\nabla_{\lambda} \Pi^{\lambda}{ }_{\nu}\right)
$$

where we have defined the fluid expansion $\vartheta_{(p-q)}=\Gamma^{\mu \nu} \nabla_{\mu} u_{\nu}$ in the $\mathrm{SO}(p-q)$ sector as well as the fluid acceleration $\mathfrak{a}^{\mu}=u^{\lambda} \nabla_{\lambda} u^{\mu}$. We now look at the charge conservation equation in directions orthogonal to the current. Let us introduce $(p-q)$ one-forms $\gamma_{\mu}^{a}$ orthogonal to $u^{\mu}$ and $\Pi^{\mu \nu}$ and mutually orthonormal. Contracting the conservation equation with $\gamma_{\mu}^{a}$, we obtain

$$
\Xi^{\lambda \mu}\left(\mathrm{d} \gamma^{a}\right)_{\mu \nu} \Xi^{\nu \rho}=0
$$

By virtue of Frobenius' theorem, the set of one-forms $\gamma^{a}$ are thus surface-forming, that is, in each point there exists an integral $(q+1)$-dimensional submanifold of the vectors $u$ and $v^{i}$. It follows that $\mathcal{M}$ is foliated into a set of $(q+1)$-dimensional submanifolds which can essentially be thought of as the level-surfaces for the dipole charge $Q$. Furthermore, the induced metric on these submanifolds is $\Xi_{\mu \nu}$ and the volume-form is precisely the structure we previously denoted by $\operatorname{Vol}_{(q+1)}$ in (2.1)-(2.2). By projecting (2.14) along $u$ and $v^{i}$ one finds the set of equations

$$
\Gamma_{\lambda}^{\nu}\left(v_{i}^{\mu} \nabla_{\mu} v_{j}^{\lambda}-v_{j}^{\mu} \nabla_{\mu} v_{i}^{\lambda}\right)=\Gamma_{\lambda}^{\nu}\left(u^{\mu} \nabla_{\mu} v_{i}^{\lambda}-v_{i}^{\mu} \nabla_{\mu} u^{\lambda}\right)=0
$$

These equations will become important when analysing equilibrium configurations.

We now consider the conservation of the stress tensor (2.10) at ideal order and project it along the $u^{\mu}, \Pi^{\mu \nu}$ and $\Gamma^{\mu \nu}$ directions, obtaining the three equations of motion

$$
\begin{aligned}
u^{\mu} \nabla_{\mu} E & =-(E+P) \nabla_{\mu} u^{\mu}+\mu Q \vartheta_{q}, \\
(E+P) \Pi_{\mu}{ }^{\nu} \mathfrak{a}_{\nu} & =\mu Q \Pi_{\mu}{ }^{\nu} \nabla_{\lambda} \Pi^{\lambda}{ }_{\nu}-\Pi_{\mu}{ }^{\nu} \nabla_{\nu}(P-\mu Q), \\
(E+P) \Gamma_{\mu}{ }^{\nu} \mathfrak{a}_{\nu} & =\mu Q \Gamma_{\mu}{ }^{\nu} \nabla_{\lambda} \Pi^{\lambda}{ }_{\nu}-\Gamma_{\mu}{ }^{\nu} \nabla_{\nu} P+\Gamma_{\mu \nu} F^{\nu},
\end{aligned}
$$

where we have defined the fluid expansion $\vartheta_{q}=\Pi^{\mu \nu} \nabla_{\mu} u_{\nu}$ in the $\mathrm{SO}(q)$ sector. Using the charge conservation equations (2.13) and the thermodynamic properties (2.6)-(2.9), we may rewrite the stress conservation equations (2.16) as the conservation of entropy current 
and Euler force equations ${ }^{7}$

$$
\begin{aligned}
\nabla_{\mu}\left(S u^{\mu}\right) & =0, \\
\Pi_{\mu}{ }^{\nu}\left(\mathfrak{a}_{\nu}+\frac{1}{T} \nabla_{\nu} T\right) & =0, \\
(E+P) \Gamma_{\mu}{ }^{\nu}\left(\mathfrak{a}_{\nu}+\frac{1}{T} \nabla_{\nu} T\right)+Q T \Gamma_{\mu}{ }^{\nu}\left(\nabla_{\nu}\left(\frac{\mu}{T}\right)-\frac{\mu}{T} \nabla_{\lambda} \Pi^{\lambda}{ }_{\nu}-\frac{1}{T} E_{\nu}\right) & =0,
\end{aligned}
$$

where $E_{\mu}=\frac{1}{q !} u^{\mu_{1}} \mathrm{Vol}_{q}^{\mu_{2} \ldots \mu_{q+1}} F_{\mu \mu_{1} \ldots \mu_{q}+1}$.

It is instructive to perform an explicit counting of independent degrees of freedom and the number of dynamical equations determining the time evolution of a given fluid configuration. There are $p$ independent components of the fluid velocity $u^{\mu}$, as one component is fixed by the normalisation condition $u^{\mu} u_{\mu}=-1$, and one degree of freedom associated with the temperature $T$. Corresponding to these $(p+1)$ degrees of freedom are $(p+1)$ dynamical equations provided by the stress tensor conservation equation (2.17). In addition, there are $(p-q) q$ independent components of $v_{\mu}^{i}$ and one degree of freedom associated with the chemical potential $\mu .{ }^{8}$ Their dynamics is provided by the charge conservation equations with a time-derivative, i.e. the first equation in eq. (2.13) and the second equation in eq. (2.15) with $(p-q) q$ components. Therefore there is a match between the number of independent degrees of freedom and the number of dynamical equations. This continues to hold at higher order in derivatives since, as we will show below, it is always possible to choose a fluid frame for which the corrections to the current $\delta J$ satisfy $\imath_{u} \delta J=0$. The remaining equations in (2.13) and (2.15) provide consistency requirements for the initial conditions on a Cauchy slice.

We will return to all these conservation equations in section 4 where we discuss equilibrium configurations. In the following section we formulate the theory at first order in derivatives.

\section{$3 \quad$ Dissipative fluids with $q$-form symmetry}

Having defined the hydrodynamics of an ideal anisotropic fluid, we now explain how to include derivative corrections. Here we shall follow the approach to relativistic dissipative hydrodynamics originally introduced by Landau and Lifshitz [31]. This approach entails postulating the existence of an entropy current $S^{\mu}$ which to any given order is constructed from the available hydrodynamic operators. The entropy current is then constrained onshell by an ultra-local version of the second law of thermodynamics, namely, $\nabla_{\mu} S^{\mu} \geq 0$. In

${ }^{7}$ The last equation in (2.17) can also be written as

$$
S T \Gamma_{\mu}{ }^{\nu}\left(\mathfrak{a}_{\nu}+\nabla_{\nu} \ln T\right)-Q \mu \Gamma_{\mu}{ }^{\nu}\left(\mathcal{K}_{\nu}-\nabla_{\nu} \ln \mu\right)=\Gamma_{\mu \nu} F^{\nu},
$$

where $\mathcal{K}^{\mu}$ is the mean extrinsic curvature of the $q$-brane embedded into the $(p+1)$-dimensional space, defined as $\mathcal{K}^{\mu}=\Xi^{\nu \lambda} \nabla_{\nu} \Xi_{\lambda}{ }^{\mu}$. This is a higher-form generalisation of the analogous equation derived for $q=1$ and $F^{\mu}=0$ in [24].

${ }^{8}$ The $q$ one-forms have $q(p+1)$ components but there are $q(q+1) / 2$ mutual orthonormality conditions, $q$ vanishing inner products with $u^{\mu}$ and $q(q-1) / 2$ components which are not independent due to $\operatorname{SO}(q)$ symmetry. This leads to $(p-q) q$ independent components. 
this section we first comment on possible choices of fluid frames and then, after picking a higher-form generalisation of the Landau frame, impose the second law of thermodynamics leading to $q+8$ transport coefficients for $q>1$ and 8 transport coefficients for $q=1$. This is followed by a derivation of Kubo formulae, which, when combined with Onsager's relation further constrains the transport coefficients, reducing the total number of transport coefficients by one. Finally, we study the constraints on the transport coefficients in different isotropic limits discussed in section 2.1.

\subsection{Dissipative corrections and choices of fluid frames}

In a derivative expansion, all the hydrodynamic currents are corrected in powers of the expansion parameter such that the total stress tensor $T^{\mu \nu}$, charge current $J^{\mu}$ and entropy current $S^{\mu}$ can be written as

$$
T^{\mu \nu}=T_{\text {ideal }}^{\mu \nu}+\delta T^{\mu \nu}+\mathcal{O}\left(\partial^{2}\right), \quad J=J_{\text {ideal }}+\delta J+\mathcal{O}\left(\partial^{2}\right), \quad S^{\mu}=S_{\text {ideal }}^{\mu}+\delta S^{\mu}+\mathcal{O}\left(\partial^{2}\right),
$$

where $\delta T^{\mu \nu}, \delta J$ and $\delta S^{\mu}$ are $\mathcal{O}(\partial)$ derivative corrections whose general form we seek to find. We decompose the corrections to these currents according to the available symmetries

$$
\begin{aligned}
\delta T^{\mu \nu} & =\alpha u^{\mu} u^{\nu}+2\left(u^{(\mu} \phi_{\Pi}^{\nu)}+u^{(\mu} \phi_{\Gamma}^{\nu)}\right)+\tau_{\Pi} \Pi^{\mu \nu}+\tau_{\Gamma} \Gamma^{\mu \nu}+\tau^{\mu \nu}, \\
\delta J & =\beta \operatorname{Vol}_{(q+1)}+\psi \wedge \operatorname{Vol}_{q}+\Upsilon, \\
\delta S^{\mu} & =\frac{1}{T} \phi_{\Pi}^{\mu}+\frac{1}{T} \phi_{\Gamma}^{\mu}-\frac{\mu}{T} \psi^{\mu}+\delta S_{\text {non-can }}^{\mu} .
\end{aligned}
$$

In (3.2) we have introduced the first order vector $\phi_{\Pi}^{\mu}$ describing the heat flux along the $\Pi^{\mu \nu}$ directions, subject to the constraints $u_{\mu} \phi_{\Pi}^{\mu}=\Gamma_{\mu \nu} \phi_{\Pi}^{\nu}=0$. Similarly, $\phi_{\Gamma}^{\mu}$ is a heat flux vector along $\Gamma^{\mu \nu}$ satisfying $u_{\mu} \phi_{\Pi}^{\mu}=\Pi_{\mu \nu} \phi_{\Gamma}^{\nu}=0$. We have also introduced $\tau_{\Pi}=\Pi_{\mu \nu} \delta T^{\mu \nu}$ and $\tau_{\Gamma}=\Gamma_{\mu \nu} \delta T^{\mu \nu}$ which denote the trace of $\delta T^{\mu \nu}$ along the $\Pi_{\mu \nu}$ and $\Gamma_{\mu \nu}$ directions respectively. Furthermore, $\tau^{\mu \nu}$ is a symmetric and traceless tensor subject to the constraints $u_{\mu} \tau^{\mu \nu}=\Pi_{\mu \nu} \tau^{\mu \nu}=\Gamma_{\mu \nu} \tau^{\mu \nu}=0$. In the decomposition of the charge current, we introduced the one-form $\psi_{\mu}$ such that $u^{\mu} \psi_{\mu}=\Pi^{\mu \nu} \psi_{\nu}=0$. In addition, $\Upsilon$ is a $(q+1)$-form orthogonal to $\delta J$, that is, $\operatorname{Vol}_{q} \wedge \star \Upsilon=0$. The scalars $\alpha$ and $\beta$ are composed of linear combinations of the one-derivative hydrodynamic scalars available, namely $\vartheta_{(p-q)}$ and $\vartheta_{q}$.

The first three terms in the entropy current are made out of vector structures which are already present in the stress tensor and charge current, and are commonly referred to as the canonical entropy current. The last term $\delta S_{\text {non-can }}^{\mu}$ is referred to as the non-canonical entropy current and accounts for independent tensor structures that can appear in the entropy current. As we shall see, up to first order in derivatives and when restricted to the parity-even sector, $\delta S_{\text {non-can }}^{\mu}$ is forced to vanish due to the second law of thermodynamics. ${ }^{9}$ Therefore the entropy current at first order in derivatives takes the canonical form. Nevertheless, we include this term here in order to facilitate the analysis of the hydrostatic sector of the theory.

\footnotetext{
${ }^{9}$ For $q=1$ we further require charge conjugation invariance, implying that terms appearing in the constitutive relations must be invariant under the transformation $v_{1}^{\mu} \rightarrow-v_{1}^{\mu}$. Relaxing parity and chargeconjugation invariance leads to many more transport coefficients. In the case of $q=1$, many of these have been written down in [19]. In a future publication, we will revisit this case in further detail [28].
} 


\subsubsection{Fluid frames}

So far, the discussion has been rather general and the decomposition and constraints have been imposed on symmetry grounds and to match the anticipated number of degrees of freedom. However, as usual, one must specify a fluid frame due to the freedom of redefining the fluid variables $T, \mu, u^{\mu}, v_{\mu}^{i}$ according to

$$
T \rightarrow T+\bar{\delta} T, \quad \mu \rightarrow \mu+\bar{\delta} \mu, \quad u \rightarrow u^{\mu}+\bar{\delta} u^{\mu}, \quad v^{i} \rightarrow v_{\mu}^{i}+\bar{\delta} v_{\mu}^{i},
$$

subjected to the orthogonality conditions

$$
u_{\mu} \bar{\delta} u^{\mu}=0, \quad u^{\mu} \bar{\delta} v_{\mu}^{i}+v_{\mu}^{i} \bar{\delta} u^{\mu}=0, \quad v^{i \mu} \bar{\delta} v_{\mu}^{j}+v^{j \mu} \bar{\delta} v_{\mu}^{i}=0 \quad \forall i, j=1, \ldots, q .
$$

This frame transformation leads to the following change in the stress tensor and charge current

$$
\begin{aligned}
\bar{\delta} T^{\mu \nu} & =\bar{\delta} E u^{\mu} u^{\nu}+\bar{\delta} P_{q} \Pi^{\mu \nu}+\bar{\delta} P \Gamma^{\mu \nu}+2(E+P) u^{(\mu} \bar{\delta} u^{\nu)}-2 \mu Q \delta^{i j} v_{i}^{(\mu} \bar{\delta} v_{j}^{\nu)}, \\
\bar{\delta} J & =\bar{\delta} Q \operatorname{Vol}_{(q+1)}+Q \bar{\delta} u \wedge \operatorname{Vol}_{q}+Q u \wedge \sum_{i=1}^{q}\left(v_{v^{i}} \operatorname{Vol}_{q}\right) \wedge \bar{\delta} v^{i} .
\end{aligned}
$$

For a fluid with particle charge $(q=0)$ a convenient choice of frame is the Landau frame defined by the conditions $u_{\mu} \delta T^{\mu \nu}=u_{\mu} \delta J^{\mu}=0$. For arbitrary $p$ and $q$, the obvious generalisation of the Landau conditions, which reduces to the Landau frame in the isotropic limits $(q=0$ or $q=p$ or $Q=0)$, is defined by

$$
u_{\mu} \delta T^{\mu \nu}=0, \quad \Pi_{\mu \lambda} \Gamma_{\nu \rho} \delta T^{\mu \nu}=0, \quad \star\left(\star \operatorname{Vol}_{(q+1)} \wedge \delta J\right)=0 .
$$

This choice of frame implies that $\alpha=\beta=\phi_{\Pi}=\phi_{\Gamma}=0$ and that $\tau_{\mu \nu}$ satisfies the orthogonality condition $\Pi_{\mu \lambda} \Gamma_{\nu \rho} \tau^{\mu \nu}=0$.

Another common frame used in the context of dissipative fluids with particle charge is the Eckart frame, defined as $u_{\mu} u_{\nu} \delta T^{\mu \nu}=\delta J^{\mu}=0$. We note that in the general case of a fluid charged under a $(q+1)$-form current, there is no direct analogue of the Eckart frame in the sense that it is not possible to set $\delta J=0$ since there is no frame transformation (3.5) that can eliminate the orthogonal components of the current $\Gamma_{\nu_{1}}{ }^{\mu_{1}} \ldots \Gamma_{\nu_{q+1}}{ }^{\mu_{q+1}} J^{\nu_{1} \ldots \nu_{q+1}}$. The closest analogue of the Eckart frame is defined by the conditions

$$
u_{\mu} u_{\nu} \delta T^{\mu \nu}=0, \quad u_{\mu} \Pi_{\nu \lambda} \delta T^{\mu \lambda}=0, \quad \star\left(\operatorname{Vol}_{q} \wedge \star \delta J\right)=0, \quad \imath_{u} \delta J=0 .
$$

This frame implies that $\alpha=\beta=\phi_{\Pi}=\psi=0$ and the constraint $\imath_{u} \Upsilon=0$. This in turn has the consequence that the interpretation given by eq. (2.14) of $\mathcal{M}$ being foliated into a set of $(q+1)$-dimensional submanifolds is in general lost, though it is recovered in the hydrostatic sector, as we shall see in section 4 . In the core of this paper we have choosen to use the Landau frame (3.6) but in appendix A we present the results of the next section in the frame of $[18,19]$, which is different than the frame (3.6). 


\subsection{Entropy current constraints}

We are now ready to obtain the constraints following from the second law of thermodynamics. To begin with, we evaluate the divergence of the entropy current given in (3.2) without making reference to a particular hydrodynamic frame. After a bit of algebra, we obtain

$$
\begin{aligned}
\nabla_{\mu} S^{\mu}= & -\frac{1}{T^{2}} \alpha u^{\mu} \nabla_{\mu} T-\frac{1}{T}\left(\phi_{\Pi}^{\mu}+\phi_{\Gamma}^{\mu}\right)\left(\frac{1}{T} \nabla_{\mu} T+\mathfrak{a}_{\mu}\right) \\
& -\frac{\tau_{\Gamma}}{T} \vartheta_{(p-q)}-\frac{\tau_{\Pi}}{T} \vartheta_{q}-\frac{1}{T} \tau^{\mu \nu} \nabla_{\mu} u_{\nu} \\
& -\beta u^{\mu} \nabla_{\mu}\left(\frac{\mu}{T}\right)-\psi^{\mu}\left(\partial_{\mu}\left(\frac{\mu}{T}\right)-\frac{\mu}{T} \nabla_{\nu} \Pi^{\nu}{ }_{\mu}-\frac{1}{T} E_{\mu}\right)-\frac{1}{T} \star\left[\star \Upsilon \wedge\left(\mu \mathrm{dVol}_{q}+\iota_{u} F\right)\right] \\
& +\nabla_{\mu} \delta S_{\text {non-can }}^{\mu},
\end{aligned}
$$

where we have defined the interior product of $(q+2)$-form field strength as $\left(\imath_{u} F\right)_{\mu_{1} \ldots \mu_{q+1}}=$ $u^{\mu} F_{\mu \mu_{1} \ldots \mu_{q+1}}$. Given our choice of fluid frame (3.6), these expressions can be simplified to

$$
\begin{aligned}
\nabla_{\mu} S^{\mu}= & -\psi^{\mu}\left(\partial_{\mu}\left(\frac{\mu}{T}\right)-\frac{\mu}{T} \nabla_{\nu} \Pi^{\nu}{ }_{\mu}-\frac{1}{T} E_{\mu}\right)-\frac{1}{T} \star\left[\star \Upsilon \wedge\left(\mu \mathrm{dVol}_{q}+\imath_{u} F\right)\right] \\
& -\frac{1}{T} \tau^{\mu \nu} \nabla_{\mu} u_{\nu}-\frac{\tau_{\Gamma}}{T} \vartheta_{(p-q)}-\frac{\tau_{\Pi}}{T} \vartheta_{q}+\nabla_{\mu} \delta S_{\text {non-can }}^{\mu} .
\end{aligned}
$$

Our main task now is to make this expression manifestly positive semi-definite for any hydrodynamic configuration. We begin by focusing on the non-canonical piece. Note that every term other than $\nabla_{\mu} \delta S_{\text {non-can }}^{\mu}$ is a product of two one-derivative tensor structures. Therefore, to ensure positive definiteness, we must ensure that $\nabla_{\mu} \delta S_{\text {non-can }}^{\mu}$ does not contain any pure two derivative terms. By an explicit counting, it is possible to show that no such terms can appear in $\delta S_{\text {non-can }}^{\mu}$ when imposing parity-invariance (and charge-

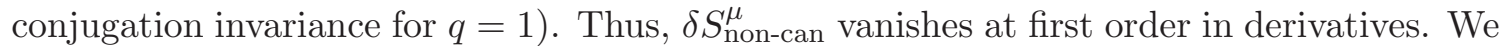
now analyse the remaining terms, proceeding term-by-term.

The first term in (3.9) implies that

$$
\psi_{\mu}=-\mathfrak{D} T h_{\{\mu\}}, \quad\{h\}=h_{\{\mu\}} d x^{\mu}=\Gamma_{\mu}{ }^{\nu}\left(\nabla_{\nu}\left(\frac{\mu}{T}\right)-\frac{\mu}{T} \nabla_{\lambda} \Pi^{\lambda}{ }_{\nu}-\frac{1}{T} E_{\nu}\right) d x^{\mu},
$$

where the function $\mathfrak{D}(T, \mu)$ satisfies $\mathfrak{D}(T, \mu) \geq 0$ and where curly brackets denote projection onto $\Gamma_{\mu \nu}$. The vector $\psi^{\mu}$ is the usual charge diffusion vector with an associated diffusion constant $\mathfrak{D}$ (Fick's diffusion law). As expected, the diffusion flux is only sensitive to the thermodynamic charges orthogonal to the current. This means that the diffusion vector vanishes in the case $q=p$, as it should [26,32].

We now consider the second term in (3.9), which leads to transport coefficients that have no analogue in fluids carrying particle charge. The simplest way to make this term positive semi-definite is by taking $\Upsilon \sim \mu \mathrm{dVol}_{q}+\imath_{u} F$, however, this is not consistent with the constraint $\operatorname{Vol}_{q} \wedge \star \Upsilon=0$. We therefore need to project $\mu \mathrm{dVol}_{q}+\imath_{u} F$ against $\operatorname{Vol}_{q}$. Since $\mu \mathrm{dVol}_{q}+\imath_{u} F$ is a $(q+1)$-form as opposed to $\operatorname{Vol}_{q}$ which is a $q$-form, we need to project at least two indices of $\mu \mathrm{dVol}_{q}+\imath_{u} F$ along the $u^{\mu}$ or $\Gamma^{\mu \nu}$ directions. Let us first define

$$
\begin{aligned}
&\left(\mathrm{dVol}_{q}^{\Gamma}\right)_{\mu_{1} \ldots \mu_{q+1}} \equiv \Gamma_{\left[\mu_{1}\right.}^{\mu} \Gamma_{\mu_{2}}^{\nu}\left(\mathrm{dVol}_{q}\right)_{\left.|\mu \nu| \mu_{3} \ldots \mu_{q+1}\right]}, \\
&\left(\mathrm{dVol}_{q}^{U}\right)_{\mu_{1} \ldots \mu_{q+1}} \equiv u^{\mu} u_{\left[\mu_{1}\right.} \Gamma_{\mu_{2}}^{\nu}\left(\mathrm{dVol}_{q}\right)_{\left.|\mu \nu| \mu_{3} \ldots \mu_{q+1}\right]} .
\end{aligned}
$$


Note that due to the definition of $\mathrm{Vol}_{q}$ in (2.2), contracting more than two of its indices along $u^{\mu}$ or $\Gamma^{\mu \nu}$ leads to zero. In light of this, (3.11) are the most generic contractions of $\mathrm{dVol}_{q}$ along $u^{\mu}$ and $\Gamma^{\mu \nu}$. Since the contraction of $\imath_{u} F$ with $u^{\mu}$ vanishes, the projection of $\mu \mathrm{dVol}_{q}+\imath_{u} F$ along one $u^{\mu}$ and one $\Gamma^{\mu \nu}$ direction leads to $\mu \mathrm{dVol}_{q}^{U}$. The remaining independent projections on the other hand are

$$
\mu \mathrm{dVol}_{q}^{\Gamma}+\Gamma^{2}\left(\imath_{u} F\right), \quad \Gamma^{I+1}\left(\imath_{u} F\right) \text { for } 2 \leq I \leq q,
$$

where $\Gamma^{I+1}\left(\imath_{u} F\right)$ has $I+1$ of its indices contracted along $\Gamma^{\mu \nu}$ and the remaining along $\Pi^{\mu \nu}$. The number of such projections is bounded by $p-q$, i.e. the number of independent $\Gamma^{\mu \nu}$ directions available. Having isolated the tensor structures, we can write down the most generic expression for $\Upsilon$ as

$$
\Upsilon=-\Theta \mathrm{dVol}_{q}^{U}-\Omega_{1}\left(\mu \mathrm{dVol}_{q}^{\Gamma}+\Gamma^{2}\left(\imath_{u} F\right)\right)-\sum_{I=2}^{q} \Omega_{I} \Gamma^{I+1}\left(\imath_{u} F\right),
$$

where $\Theta(T, \mu)$ and $\Omega_{I}(T, \mu)$ satisfy $\Theta(T, \mu), \Omega_{I}(T, \mu) \geq 0$ and are new types of current resistivities that appear for fluids carrying $q$-brane charge.

We now look at the corrections due to non-trivial terms in the stress tensor. We start by looking at the term involving $\tau_{\mu \nu}$. To this end we decompose the covariant derivative of the fluid velocity according to

$$
\nabla_{\mu} u_{\nu}=-u_{\mu} \mathfrak{a}_{\nu}+\omega_{\mu \nu}+\frac{\vartheta_{q}}{q} \Pi_{\mu \nu}+\frac{\vartheta_{(p-q)}}{p-q} \Gamma_{\mu \nu}+\Sigma_{\mu \nu} .
$$

Here $\omega_{\mu \nu}=\Delta_{\mu}{ }^{\lambda} \Delta_{\nu}{ }^{\rho} \nabla_{[\lambda} u_{\rho]}$ is the vorticity of the fluid while $\Sigma_{\mu \nu}$ is the symmetric and traceless part of $\nabla_{\mu} u_{\nu}$ orthogonal to $u$ decomposed according to the available SO-sectors such that ${ }^{10}$

$$
\Sigma_{\mu \nu}=\frac{1}{2}\left(\mathcal{L}_{u} \Gamma_{\{\mu \nu\}}+\mathcal{L}_{u} \Pi_{\langle\mu \nu\rangle}\right)+\mathcal{L}_{u} \Gamma_{\{(\mu \nu)\rangle}+\mathcal{L}_{u} \Pi_{\{(\mu \nu)\rangle} .
$$

Here $\mathcal{L}_{u}$ denotes the Lie derivative with respect to $u^{\mu}$ and the curly (angled) brackets denote projection onto $\Gamma_{\mu \nu}\left(\Pi_{\mu \nu}\right)$ and subtraction of the trace. For example

$$
\mathcal{L}_{u} \Gamma_{\{\mu \nu\}}=\Gamma_{\mu}^{\lambda} \Gamma_{\nu}^{\rho}\left(u^{\alpha} \nabla_{\alpha} \Gamma_{\lambda \rho}+\Gamma_{\alpha \rho} \nabla_{\lambda} u^{\alpha}+\Gamma_{\lambda \alpha} \nabla_{\rho} u^{\alpha}-\frac{2}{p-q} \Gamma_{\lambda \rho} \Gamma^{\alpha \beta} \nabla_{\alpha} u_{\beta}\right) .
$$

Using (3.15), we see that it is possible to add three distinct terms to $\tau_{\mu \nu}: \mathcal{L}_{u} \Pi_{\langle\mu \nu\rangle}, \mathcal{L}_{u} \Gamma_{\{\mu \nu\}}$ and $\mathcal{L}_{u} \Gamma_{\{(\mu \nu)\rangle}+\mathcal{L}_{u} \Pi_{\{(\mu \nu)\rangle}$. Note that via projections of $\nabla_{\mu} u_{\nu}$ only the linear combination of $\mathcal{L}_{u} \Gamma_{\{(\mu \nu)\rangle}$ and $\mathcal{L}_{u} \Pi_{\{(\mu \nu)\rangle}$ provides an independent tensor structure. Therefore $\tau_{\mu \nu}$ can only depend on this sum. In any case, the second Landau condition in (3.6) eliminates a possible $\mathcal{L}_{u} \Gamma_{\{(\mu \nu)\rangle}+\mathcal{L}_{u} \Pi_{\{(\mu \nu)\rangle}$ term in $\tau^{\mu \nu}$. This implies that in this frame there is no shear cross-viscosity between the two sectors. Therefore, we are lead to take

$$
\tau_{\mu \nu}=-\eta_{\Pi} \mathcal{L}_{u} \Pi_{\langle\mu \nu\rangle}-\eta_{\Gamma} \mathcal{L}_{\mu} \Gamma_{\{\mu \nu\}},
$$

\footnotetext{
${ }^{10}$ For the purposes of comparison, note that for an isotropic fluid the decomposition (3.14) may be expressed as

$$
\nabla_{\mu} u_{\nu}=-u_{\mu} \mathfrak{a}_{\nu}+\omega_{\mu \nu}+\frac{\vartheta_{p}}{p} \Delta_{\mu \nu}+\frac{1}{2} \Delta_{\mu}{ }^{\lambda} \Delta_{\nu}{ }^{\rho}\left(\mathcal{L}_{u} g_{\lambda \rho}-\frac{1}{p} g_{\lambda \rho} \operatorname{Tr} \mathcal{L}_{u} g\right)
$$
}


where $\eta_{\Pi}(T, \mu)$ and $\eta_{\Gamma}(T, \mu)$ satisfy $\eta_{\Pi}(T, \mu), \eta_{\Gamma}(T, \mu) \geq 0$ and denote the shear viscosity in the $\mathrm{SO}(q)$ and $\mathrm{SO}(p-q)$ sectors, respectively.

Finally, we look at the $\tau_{\Pi}$ and $\tau_{\Gamma}$ terms in (3.9). The most general ansatz for these terms is given in terms of first order scalars such that

$$
\tau_{\Pi}=-q\left(\zeta_{\Pi} \vartheta_{q}+\zeta_{\Pi, \Gamma} \vartheta_{(p-q)}\right), \quad \tau_{\Gamma}=-(p-q)\left(\zeta_{\Gamma} \vartheta_{(p-q)}+\zeta_{\Gamma, \Pi} \vartheta_{q}\right) .
$$

Here $\zeta_{\Pi}$ is a bulk viscosity in the $\mathrm{SO}(q)$ sector, $\zeta_{\Gamma}$ a bulk viscosity in the $\mathrm{SO}(p-q)$ sector and $\zeta_{\Pi, \Gamma}, \zeta_{\Gamma, \Pi}$ are two bulk cross-viscosities. This ansatz, when introduced into (3.9) leads to the following contribution to the divergence of the entropy currrent

$$
\nabla_{\mu} S^{\mu} \sim \frac{1}{T}\left(\begin{array}{c}
\vartheta_{(p-q)} \\
\vartheta_{q}
\end{array}\right)^{\mathrm{T}}\left(\begin{array}{cc}
(p-q) \zeta_{\Gamma} & (p-q) \zeta_{\Gamma, \Pi} \\
q \zeta_{\Pi, \Gamma} & q \zeta_{\Pi}
\end{array}\right)\left(\begin{array}{c}
\vartheta_{(p-q)} \\
\vartheta_{q}
\end{array}\right) .
$$

Requiring this quadratic form to be positive semi-definite implies that we must have

$$
\zeta_{\Gamma} \geq 0, \quad \zeta_{\Pi} \geq 0, \quad q(p-q) \zeta_{\Gamma} \zeta_{\Pi} \geq \frac{1}{4}\left((p-q) \zeta_{\Gamma, \Pi}+q \zeta_{\Pi, \Gamma}\right)^{2} .
$$

This completes the requirements of the second law of thermodynamics.

Summarising, we have the following first order derivative corrections to the stress tensor, charge and entropy current ${ }^{11}$

$$
\begin{aligned}
\delta T_{\mu \nu} & =-\eta_{\Pi} \mathcal{L}_{u} \Pi_{\langle\mu \nu\rangle}-\eta_{\Gamma} \mathcal{L}_{u} \Gamma_{\{\mu \nu\}}-\Pi_{\mu \nu}\left(\zeta_{\Pi} \vartheta_{q}+\zeta_{\Pi, \Gamma} \vartheta_{(p-q)}\right)-\Gamma_{\mu \nu}\left(\zeta_{\Gamma} \vartheta_{(p-q)}+\zeta_{\Gamma, \Pi} \vartheta_{q}\right) \\
\delta J & =-\mathfrak{D} T\{h\} \wedge \mathrm{Vol}_{q}-\Theta \mathrm{dVol}_{q}^{U}-\Omega_{1}\left(\mu \mathrm{dVol}_{q}^{\Gamma}+\Gamma^{2}\left(\imath_{u} F\right)\right)-\sum_{I=2}^{q} \Omega_{I} \Gamma^{I+1}\left(\imath_{u} F\right), \\
\delta S_{\mu} & =\mathfrak{D} \mu T h_{\{\mu\}},
\end{aligned}
$$

parametrised by $q+8$ transport coefficients for $q>1$ and 8 for $q=1$, namely, 2 shear viscosities $\eta_{\Pi}, \eta_{\Gamma}, 4$ bulk viscosities $\zeta_{\Pi}, \zeta_{\Gamma}, \zeta_{\Pi, \Gamma}, \zeta_{\Gamma, \Pi}, 1$ diffusion constant $\mathfrak{D}$ and $q+1$ kinds of resistivities $\Theta, \Omega_{I}$ satisfying

$$
\left(\eta_{\Pi}, \eta_{\Gamma}, \mathfrak{D}, \Theta, \Omega_{I}, \zeta_{\Gamma}, \zeta_{\Pi}\right) \geq 0, \quad q(p-q) \zeta_{\Gamma} \zeta_{\Pi} \geq \frac{1}{4}\left((p-q) \zeta_{\Gamma, \Pi}+q \zeta_{\Pi, \Gamma}\right)^{2} .
$$

In the next section we will show that Onsager's relation implies that the bulk crossviscosities must be equal, i.e., $\zeta_{\Pi, \Gamma}=\zeta_{\Gamma, \Pi}=\zeta$ as also was shown to be the case for $q=1$ in $[18,19]$.

It is worth noting that compared to the case $q=1$ studied in $[18,19]$, there are $q$ additional transport coefficients for $q>1$, namely the shear viscosity $\eta_{\Pi}$ in the $\operatorname{SO}(q)$ sector and $q-1$ resistivities $\Omega_{I \geq 2}$. In the case $q=1$ the shear viscosity in the $\mathrm{SO}(q)$ sector is obsolete since $\mathcal{L}_{u} \Pi_{\langle\mu \nu\rangle}=0$ as there is only one vector $v_{1}^{\mu}$.

${ }^{11}$ As earlier advertised, the total entropy current up to first order in derivatives takes the canonical form

$$
S^{\mu}=s u^{\mu}-\frac{1}{T} \delta T^{\mu \nu} u_{\nu}-\frac{\mu}{T} \delta J^{\mu \mu_{1} \ldots \mu_{q}}\left(\operatorname{Vol}_{q}\right)_{\mu_{1} \ldots \mu_{q}} .
$$




\subsection{Kubo formulae}

Here we derive Kubo formulae for the transport coefficients found above using the variational background method of [33]. The results presented here can be seen as an extension of those derived in $[18,19]$ for $q=1$, though in a different frame. These formulae, together with Onsager's relation imply a constraint between the two bulk cross-viscosities found above, leading to one less independent transport coefficient.

We split the background coordinates $x^{\alpha}$ into the set $t, y^{a}, z^{i}$ where $t$ is the time coordinate, $z^{i}$ with $i=1, \ldots, q$ label the $q$ directions along $v_{i}^{\mu}$, and $y^{a}$ with $a=1, \ldots, p-q$ label the orthogonal directions to $u^{\mu}$ and $v_{i}^{\mu}$. We consider an equilibrium fluid configuration in flat space with vanishing background gauge field $A_{q+1}$ and with velocity profile

$$
u^{\mu}=\delta^{\mu}{ }_{t}, \quad v_{i}^{\mu}=\delta^{\mu}{ }_{i} .
$$

Performing a small time-dependent, but homogeneous in space, perturbation of all fields $u^{\mu} \rightarrow u^{\mu}+\delta u^{\mu}, v_{i}^{\mu} \rightarrow v_{i}^{\mu}+\delta v_{i}{ }^{\mu}, g_{\mu \nu} \rightarrow \eta_{\mu \nu}+\delta h_{\mu \nu}$ and $A_{q+1} \rightarrow \delta A_{q+1}$ leads to

$$
\delta u^{t}=\frac{1}{2} \delta h_{t t}, \quad \delta v_{i}^{t}=\delta u^{i}+\delta h_{t i}, \quad \delta_{k(j} \delta v_{i)}{ }^{k}=-\frac{1}{2} \delta h_{i j},
$$

as well as $\nabla_{(a} u_{b)}=\partial_{t} \delta h_{a b} / 2$ and $\nabla_{(i} u_{j)}=\partial_{t} \delta h_{i j} / 2$. We define the one-point functions

$$
\mathbb{T}^{\mu \nu}=\sqrt{-g}\left\langle T^{\mu \nu}\right\rangle, \quad \mathbb{J}^{\mu_{1} \ldots \mu_{q+1}}=\sqrt{-g}\left\langle J^{\mu_{1} \ldots \mu_{q+1}}\right\rangle,
$$

which according to linear response theory can be written in terms of retarded Green's functions of frequency $\omega$ such that (see e.g. [34])

$$
\begin{aligned}
\delta \mathbb{T}^{\mu \nu}(\omega) & =\frac{1}{2} G_{T T}^{\mu \nu, \lambda \rho}(\omega) \delta h_{\lambda \rho}+\frac{1}{(q+1) !} G_{T J}^{\mu \nu, \mu_{1} \ldots \mu_{q+1}}(\omega) \delta\left(A_{q+1}\right)_{\mu_{1} \ldots \mu_{q+1}}, \\
\delta J^{\mu_{1} \ldots \mu_{q+1}}(\omega) & =\frac{1}{2} G_{J T}^{\mu_{1} \ldots \mu_{q+1}, \lambda \rho} \delta h_{\lambda \rho}(\omega)+\frac{1}{(q+1) !} G_{J J}^{\mu_{1} \ldots \mu_{q+1}, \nu_{1} \ldots \nu_{q+1}}(\omega) \delta\left(A_{q+1}\right)_{\nu_{1} \ldots \nu_{q+1}} .
\end{aligned}
$$

Using (3.24) and the form of the stress tensor and charge current (3.21) one finds

$$
\begin{aligned}
& \eta_{\Gamma}=\lim _{\omega \rightarrow 0} \frac{1}{\omega} \operatorname{Im} G_{T T}^{a b, a b}, \text { for } a \neq b, \\
& \eta_{\Pi}=\lim _{\omega \rightarrow 0} \frac{1}{\omega} \operatorname{Im} G_{T T}^{i j, i j}, \quad \text { for } i \neq j, \quad \zeta_{\Gamma}+2 \frac{(p-q-1)}{(p-q)} \eta_{\Gamma}=\lim _{\omega \rightarrow 0} \frac{1}{\omega} \operatorname{Im} G_{T T}^{a a, a a}, \\
& \zeta=\zeta_{\Pi, \Gamma}=\zeta_{\Gamma, \Pi}=\lim _{\omega \rightarrow 0} \frac{1}{\omega} \operatorname{Im} G_{T T}^{i i, a a}=\lim _{\omega \rightarrow 0} \frac{1}{\omega} \operatorname{Im} G_{T T}^{a a, i i}, \\
& \mathfrak{D}=\lim _{\omega \rightarrow 0} \frac{1}{\omega} \operatorname{Im} G_{J J}^{a i_{1} \ldots i_{q}, a i_{1} \ldots i_{q}}, \\
& \Omega_{I}=\lim _{\omega \rightarrow 0} \frac{1}{\omega} \operatorname{Im} G_{J J}^{a_{1} \ldots a_{I+1} i_{1} \ldots i_{q-I}, a_{1} \ldots a_{I+1} i_{1} \ldots i_{q-I}}, \\
& \Theta=\lim _{\omega \rightarrow 0} \frac{1}{\omega} \operatorname{Im} G_{T T}^{i i, i i}, \\
& \operatorname{Im} G_{J T}^{a t i_{1} \ldots i_{q-1}, a i} .
\end{aligned}
$$

Note that in the third line we have identified $\zeta=\zeta_{\Pi, \Gamma}=\zeta_{\Gamma, \Pi}$. This follows from Onsager's relation for mixed correlation functions, exactly in the same manner as in $[18,19]$ for $q=1$. This implies that parity-even fluids with $q$-form symmetry are characterised by $q+7$ independent transport coefficients at first order in derivatives for $q>1$ and 7 independent transport coefficients for $q=1$. 


\subsection{Constraints on transport in the isotropic limits}

As stressed in section 2, the hydrodynamics of fluids with $q$-form symmetry must reduce to the hydrodynamics of (charged) isotropic fluids in the limits $q=0, q=p$ or $Q \rightarrow 0$. This requirement imposes relations between transport coefficients in the isotropic limit. The corrections to the stress tensor and charge current (3.21) in the isotropic limit must take the following form in the Landau frame (see e.g. $[26,32]$ )

$$
\begin{aligned}
\delta T^{\mu \nu} & =-\eta \Delta_{\mu}{ }^{\lambda} \Delta_{\nu}{ }^{\rho}\left(\mathcal{L}_{u} g_{\lambda \rho}-\frac{1}{p} g_{\lambda \rho} \operatorname{Tr} \mathcal{L}_{u} g\right)-\xi \vartheta_{p} \Delta^{\mu \nu}, \\
\delta J^{\mu} & =-\mathcal{D} T \Delta^{\mu \nu}\left(\nabla_{\nu}\left(\frac{\mu}{T}\right)-\frac{1}{T} E_{\nu}\right),
\end{aligned}
$$

for some coefficients $\eta, \xi, \mathcal{D}$ and where we have only considered corrections to the current for the $q=0$ case since the total current vanishes in the limit $Q \rightarrow 0$ while the corrections $\delta J$ vanish when $q=p$ since in this case $Q$ is a global charge and hence non-dynamical.

The most non-trivial limit is the uncharged (neutral) limit $Q \rightarrow 0$ which must remove all sources of anisotropy. This means that when rewriting the stress tensor in (3.21) in terms of a $\mathrm{SO}(p)$ and a $\mathrm{SO}(q)$ sector, the $\mathrm{SO}(q)$ sector must vanish in the limit $Q \rightarrow 0$. This imposes non-trivial constraints on the transport coefficients in this limit. In particular we must have

$$
\eta_{\Pi}^{(0)}=\eta_{\Gamma}^{(0)}, \quad \zeta^{(0)}=\frac{(p-q) \zeta_{\Gamma}^{(0)}-q \zeta_{\Pi}^{(0)}}{p-2 q}
$$

where the subscript (0) denotes the value of the transport coefficients in the limit $Q \rightarrow 0$. In addition to (3.28) one also obtains

$$
\zeta_{\Gamma}^{(0)}-\zeta^{(0)}-2 \frac{\eta_{\Gamma}^{(0)}}{(p-q)}=0, \quad \zeta^{(0)}-\left(\zeta_{\Pi}^{(0)}+\frac{1}{2} \zeta_{\Gamma}^{(0)}\right)+\frac{p}{q(p-q)} \eta_{\Pi}^{(0)}=0 .
$$

However, the second condition above is redundant as the first condition in (3.29) together with conditions (3.28) imply the second in (3.29). Given these relations, comparison with (3.27) yields

$$
\eta=\eta^{(0)}, \quad \xi=\zeta_{\Gamma}^{(0)}-\frac{q}{p(p-q)} \eta_{\Gamma}^{(0)} .
$$

As we shall see in a companion paper where we study a specific class of gravitational duals to these fluid configurations in flat space [35], the first condition in (3.28) is actually satisfied for any value of $p, q, Q$ since it is found that $\eta_{\Pi} / S=\eta_{\Gamma} / S=1 / 4 \pi$. The second condition in (3.28) is also satisfied for all $p, q, Q$ for the same class of gravitational duals. This latter relation is also observed in the context of another class of gravitational duals in Anti-de Sitter space studied in [20], for which it was found $\zeta_{\Gamma}=\zeta_{\Pi} / 4=-\zeta / 2$ (see eq. (88) in [20]) in agreement with the second condition in $(3.28)$ for $(p, q)=(3,1)$.

In the limit $q=0$ for which the fluid is carrying particle charge, we obtain the identification

$$
\eta=\eta_{\Gamma}^{(q=0)}, \quad \xi=\zeta_{\Gamma}^{(q=0)}, \quad \mathcal{D}=\mathfrak{D}^{(q=0)},
$$

while in the limit $q=p$ we find

$$
\eta=\eta_{\Pi}^{(q=p)}, \quad \xi=\zeta_{\Pi}^{(q=p)} .
$$


Taking the subsequent limit $Q \rightarrow 0$ of the viscosities $\eta$ and $\xi$ in (3.31)-(3.32) must lead to the results (3.30) so that

$$
\eta=\left.\eta_{\Pi}^{(q=p)}\right|_{Q \rightarrow 0}=\left.\eta_{\Gamma}^{(q=0)}\right|_{Q \rightarrow 0}=\eta^{(0)}, \quad \xi=\left.\zeta_{\Pi}^{(q=p)}\right|_{Q \rightarrow 0}=\left.\zeta_{\Gamma}^{(q=0)}\right|_{Q \rightarrow 0}=\zeta_{\Gamma}^{(0)}-\frac{q}{p(p-q)} \eta_{\Gamma}^{(0)} .
$$

As will be shown in a companion publication [35], it is possible to verify these relations using a family of gravitational duals parametrised by $p, q$ and $Q$.

\section{Equilibrium partition function}

In this section we study the hydrostatic sector of the hydrodynamic theory presented in the previous sections. We show that the most general partition function that gives rise to the fluids with $q$-form symmetry introduced above, regardless of the microscopic origin of the vectors $v_{i}^{\mu}$, requires the existence of $q$ mutually commuting spacelike isometries in addition to a timelike isometry. This analysis shows that, under these assumptions, the most general partition function is a $q$-form generalisation of the free energy for $q=1$ fluids considered in [24]. At the end of this section, we show that further constraints must be imposed on the partition function in order to describe the hydrostatic sector of the theory.

\subsection{The partition function}

In order to describe equilibrium solutions, we consider the existence of a timelike Killing vector field $\mathbf{k}^{\mu}$ with modulus $\mathbf{k}=\left|-g_{\mu \nu} \mathbf{k}^{\mu} \mathbf{k}^{\nu}\right|^{1 / 2}$. For the configuration to be stationary, the Lie derivative along $\mathbf{k}^{\mu}$ of any quantity characterising the fluid must vanish, in particular

$$
\mathcal{L}_{\mathbf{k}} g_{\mu \nu}=0, \quad \mathcal{L}_{\mathbf{k}} A_{q+1}=0 .
$$

In order to construct the partition function we must classify the ideal order Lorentz and gauge invariants on which the partition may depend on. It is straightforward to realise that there are only two possible scalars that can be constructed from background data, namely

$$
\mathbf{k}^{2}, \quad \mathbf{k}^{\mu} A_{\mu} \text { for } \quad q=0
$$

where the second scalar is only defined for $q=0$. Note that under a time independent gauge transformation $\Lambda$ with $\mathbf{k}^{\mu} \partial_{\mu} \Lambda=0$, the second scalar is indeed gauge-invariant. Requiring the partition function to be dependent on these two scalars leads to the stress tensor, currents and thermodynamics of a $q=0$ charged fluid.

For $q>0$, however, there is no natural equivalent of the second scalar in (4.2) since the respective contraction $\mathbf{k}^{\mu} A_{\mu \mu_{1} \mu_{2} \ldots \mu_{q}}^{q+1}$ is a $q$-form. Consequently, to describe the hydrostatic sector of fluids with a $q$-form symmetry, we need to introduce additional tensor structures on the background. If we were provided with a $(q+1)$-dimensional Killing subspace, we could use the associated volume form to contract with the indices of $A_{\mu_{1} \mu_{2} \ldots \mu_{q+1}}^{q+1}$ and obtain a gauge-invariant scalar. To this end, we assume the existence of $q$ mutually commuting spacelike Killing vectors $\ell_{i}^{\mu}$ that satisfy $\left[\mathbf{k}^{\mu}, \ell_{i}^{\nu}\right]=0$, but whose inner product $\mathbf{k}^{\mu} \ell_{i}^{\nu} g_{\mu \nu}$ 
does not necessarily vanish. Since the vectors $\ell_{i}^{\mu}$ are Killing vectors fields, one must also have that

$$
\mathcal{L}_{\ell_{i}} g_{\mu \nu}=0, \quad \mathcal{L}_{\ell_{i}} A_{q+1}=0, \quad \mathcal{L}_{\ell_{i}} \mathbf{k}^{\mu}=0 .
$$

The subspace spanned by the vectors $\ell_{i}^{\mu}$ and $\mathbf{k}^{\mu}$ is the desired $(q+1)$-dimensional Killing subspace, which is invariant under an arbitrary redefinition of $\ell_{i}^{\mu}$ involving themselves and $\mathbf{k}^{\mu}$, i.e.

$$
\ell_{i}^{\mu} \rightarrow R_{i}^{j} \ell_{j}^{\mu}+P_{i} \mathbf{k}^{\mu}
$$

Here $R_{i}^{j}$ is an arbitrary $q \times q$ non-singular matrix and $P_{i}$ is a $q$-vector. In addition to the timelike Killing vector field $\mathbf{k}^{\mu}$, we require the equilibrium partition function to only depend on the $(q+1)$-dimensional Killing subspace, and not on the Killing vector fields $\ell_{i}^{\mu}$ individually. This is equivalent to demanding the invariance of the partition function under (4.4). As it will be shown below, this requirement leads to the correct constitutive relations for $q$-form hydrodynamics as introduced in section 2 .

It is convenient to fix a large part of the redefinition freedom (4.4) by instead working with a set of orthonormal vectors defined as

$$
\zeta_{i}^{\mu}=S_{i}^{j}\left(\ell_{j}^{\mu}-\frac{\mathbf{k}_{\nu} \ell_{j}^{\nu}}{\mathbf{k}^{2}} \mathbf{k}^{\mu}\right), \quad \text { where } \quad S_{i}{ }^{k} S_{j}{ }^{l} \ell_{k}^{\mu} \ell_{l}^{\nu}\left(g_{\mu \nu}-\frac{\mathbf{k}_{\mu} \mathbf{k}_{\nu}}{\mathbf{k}^{2}}\right)=\delta_{i j}
$$

which satisfy $\zeta_{i}^{\mu} \mathbf{k}_{\mu}=0$ and $\zeta_{i}^{\mu} \zeta_{j}^{\nu} g_{\mu \nu}=\delta_{i j}$, and span a $q$-dimensional subspace transverse to $\mathbf{k}^{\mu}$. The second condition above can be seen as determining the matrix $S_{i}^{j}$ up to a residual $\mathrm{SO}(q)$ symmetry which rotates the vectors $\zeta_{i}^{\mu}$. This is precisely the $\mathrm{SO}(q)$ symmetry of $q$ form hydrodynamics as introduced in section 2 (see footnote 4). Imposing the normalisation conditions removes $q+q(q+1) / 2$ components from $\zeta_{i}^{\mu}$, while the $\mathrm{SO}(q)$ symmetry removes further $q(q-1) / 2$ components. This leads to $(p-q) q$ independent components in $\zeta_{i}^{\mu}$, matching the counting performed in section 2 for the independent components of the fields $v_{i}^{\mu}$. As a trade-off for working in an orthonormal basis, the $\zeta_{i}^{\mu}$, s are not necessarily Killing vector fields, since while $\boldsymbol{\ell}_{i}^{\nu} \mathbf{k}_{\nu} / \mathbf{k}^{2}$ cannot depend on the directions along the timelike and spacelike isometries, it may depend on the transverse $(p-q)$ directions.

Given these considerations, it follows that there are now two scalars that can be built from background data and on which the partition function may depend on, namely ${ }^{12}$

$$
\mathbf{k}^{2}, \quad \sigma \equiv \frac{1}{q !} \epsilon^{j i_{1} \ldots i_{q-1}} \zeta_{i_{1}}^{\mu_{1}} \ldots \zeta_{i_{q-1}}^{\mu_{q-1}} \mathbf{k}^{\mu} \zeta_{j}^{\nu} A_{\mu \nu \mu_{1} \ldots \mu_{q-1}}^{q+1}
$$

which are well defined for all $p$ and $q .{ }^{13}$ In defining $\sigma$, we have introduced a $\mathrm{SO}(q)$ covariant Levi-Civita tensor with $\epsilon_{123 \ldots q}=1$. We can now consider the partition function to be a

\footnotetext{
${ }^{12}$ The scalar $\sigma$ is the pullback of the background gauge field $A_{q+1}$ onto the $(q+1)$-dimensional subspace spanned by the vectors $\mathbf{k}^{\mu}$ and $\ell_{i}^{\mu}$. This scalar is gauge invariant due to the Killing properties of $\mathbf{k}^{\mu}$ and $\ell_{i}^{\mu}$. On the other hand, zero-derivative scalars constructed from the projection of $A_{q+1}$ onto the $(p-q)$ dimensional subspace will not be gauge invariant for arbitrary $p$ and $q$.

${ }^{13}$ One may shift the scalar $\sigma$ by a constant $\mu_{0}$ which would turn out to have the interpretation of a constant global chemical potential.
} 
function of these two scalars, therefore we write ${ }^{14}$

$$
W=\frac{1}{T_{0}} \int_{\Sigma} d^{D-1} x \sqrt{-g} \mathcal{P}\left(\mathbf{k}^{2}, \sigma\right)
$$

where $T_{0}$ is the constant global temperature and the integral is taken over a spatial hypersurface $\Sigma$. From this partition function we may obtain the stress tensor and currents in the following manner

$$
\begin{aligned}
T^{\mu \nu} & =\frac{2}{\sqrt{-g}} \frac{\delta W}{\delta g_{\mu \nu}}=\mathcal{P} g^{\mu \nu}+2 \frac{\partial \mathcal{P}}{\partial \mathbf{k}^{2}} \mathbf{k}^{\mu} \mathbf{k}^{\nu}-\sigma \frac{\partial \mathcal{P}}{\partial \sigma} \delta^{i j} \zeta_{i}^{\mu} \zeta_{j}^{\nu} \\
J^{\mu_{1} \ldots \mu_{q+1}} & =\frac{1}{\sqrt{-g}} \frac{\delta W}{\delta A_{\mu_{1} \ldots \mu_{q+1}}^{q+1}}=(q+1) \frac{\partial \mathcal{P}}{\partial \sigma} \mathbf{k}^{\left[\mu_{1}\right.} \zeta_{i_{1}}^{\mu_{2}} \ldots \zeta_{i_{q}}^{\mu_{q+1]}} \epsilon^{i_{1} \ldots i_{q}}
\end{aligned}
$$

Comparing this stress tensor and current with (2.1) and (2.5) one identifies

$$
P=\mathcal{P}, \quad Q=\mathbf{k} \frac{\partial \mathcal{P}}{\partial \sigma}, \quad u^{\mu}=\frac{\mathbf{k}^{\mu}}{\mathbf{k}}, \quad \mu=\frac{\sigma}{\mathbf{k}}, \quad E+P=2 \mathbf{k}^{2} \frac{\partial \mathcal{P}}{\partial \mathbf{k}^{2}}, \quad v_{i}^{\mu}=\zeta_{i}^{\nu} .
$$

In addition, by obtaining the total entropy from the partition function

$$
S_{\mathrm{tot}}=\frac{\partial\left(T_{0} W\right)}{\partial T_{0}}=\int_{\Sigma} d^{D-1} x \sqrt{-g} \frac{\partial \mathcal{P}}{\partial T_{0}},
$$

and comparing with that obtained by integrating the ideal order entropy current (2.7) leads to the identification $T=T_{0} / \mathbf{k}$, which together with (4.9), yield the thermodynamic properties (2.9). The solution defined by (4.9) and $T=T_{0} / \mathbf{k}$ agrees with that obtained in [24] for $q=1$ which reduces to the one considered in [18] only when $\mathbf{k}_{\mu} \ell_{1}^{\mu}=0$.

\subsection{Additional constraints from the second law of thermodynamics}

In equilibrium, a hydrodynamic theory is expected not to produce any entropy. If the solution (4.9) provided by the equilibrium partition function above is truly an equilibrium solution, then not only must the ideal order fluid equations (2.13), (2.15) and (2.17) be trivially satisfied, but also the divergence of the entropy current (3.8) must vanish so that no entropy is produced. We can isolate all the independent tensor structures appearing in the entropy current divergence and first order constitutive relations in terms of

$$
\nabla_{(\mu}\left(\frac{u_{\nu)}}{T}\right), \quad \mathrm{d}\left(\frac{\mu}{T} \operatorname{Vol}_{q}\right)+\frac{1}{T} \imath_{u} F .
$$

\footnotetext{
${ }^{14}$ If we had not required the partition function to be invariant under (4.4), it could depend on many other scalars such as $f_{i}=\ell_{i}^{\mu} \mathbf{k}_{\mu}$ and $h_{i j}=\ell_{i}^{\mu} \ell_{j}^{\nu}\left(g_{\mu \nu}-\mathbf{k}_{\mu} \mathbf{k}_{\nu} / \mathbf{k}^{2}\right)$ for all $i, j=1,2, \ldots, q$, in addition to $\mathbf{k}^{2}$ and $\sigma$. Moreover, it would have also been possible to consider $\mathrm{SO}(q)$ invariant scalars that are not invariant under arbitrary redifinitions given by (4.4). This class of scalars includes $f_{i} f_{j} h^{i j}$ and det $h_{i j}$ where $h^{i j}$ is the inverse of $h_{i j}$. All these possible extra dependences, though perhaps of interest for the description of other physical systems, would be incompatible with the $q$-form fluid that we are trying to describe, whose thermodynamic properties only depend on two scalar degrees of freedom, namely, $T$ and $\mu$.
} 
All the other tensor structures appearing in section 3 are given by projections of these along $u^{\mu}, \Pi^{\mu \nu}$ and $\Gamma^{\mu \nu}$, leading to

$$
\begin{aligned}
& u^{\mu} \nabla_{\mu} T, \quad \Gamma_{\mu}{ }^{\nu}\left(\frac{1}{T} \nabla_{\nu} T+\mathfrak{a}_{\nu}\right), \quad \Pi_{\mu}{ }^{\nu}\left(\frac{1}{T} \nabla_{\nu} T+\mathfrak{a}_{\nu}\right), \quad \vartheta_{(p-q)}, \quad \vartheta_{q}, \\
& \mathcal{L}_{u} \Gamma_{\{\mu \nu\}}, \quad \mathcal{L}_{u} \Pi_{\langle\mu \nu\rangle}, \quad \mathcal{L}_{u} \Gamma_{\{(\mu \nu)\rangle}+\mathcal{L}_{u} \Pi_{\{(\mu \nu)\rangle}, \\
& u^{\mu} \nabla_{\mu}\left(\frac{\mu}{T}\right), \quad h_{\{\mu\}}, \quad \mathrm{dVol}_{q}^{U}, \quad \mu \mathrm{dVol}_{q}^{\Gamma}+\Gamma^{2}\left(\imath_{u} F\right),\left.\quad \Gamma^{I+1}\left(\imath_{u} F\right)\right|_{I=2} ^{q} .
\end{aligned}
$$

One may readily check that the solution (4.9) together with $T=T_{0} / \mathbf{k}$ leads to the vanishing of the first tensor structure in (4.11) but not the second. In components, this corresponds to the last two terms in (4.12), which do not vanish for the solution (4.9). From here it follows that all the conservation laws are trivially satisfied, however, the terms proportional to $\Omega_{I}$ in (3.21) contribute to entropy production. To remedy this situation, we must require the second term in (4.11) to vanish by hand. In equilibrium, this term evaluates to ${ }^{15}$

$$
\mathrm{d}\left(\frac{\mu}{T} \operatorname{Vol}_{q}\right)+\frac{1}{T} \imath_{u} F=\frac{1}{T_{0}} \mathrm{~d}\left(\sigma \operatorname{Vol}_{q}-\imath_{\mathbf{k}} A_{q+1}\right)=0,
$$

where we have used the identity that $\mathcal{L}_{\mathbf{k}} A_{q+1}=\mathrm{d}\left(\imath_{\mathbf{k}} A_{q+1}\right)+\imath_{\mathbf{k}} F$ vanishes in equilibrium. To have a consistent hydrostatic solution, we must require this additional condition on our hydrostatic backgrounds on top of the existence of $q$ additional spacelike isometries. ${ }^{16}$

To summarise, apart from the existence of a timelike isometry, we have introduced two additional constraints on our backgrounds so that they admit a hydrostatic solution: they must admit $q$ additional spacelike isometries and they must satisfy the constraint (4.13). This seems to be a feature of hydrodynamics with higher-form symmetries. However, one may wonder if at higher orders in the derivative expansion further conditions must be imposed on these backgrounds to ensure the consistency of the hydrostatic sector so that no entropy is produced. Extending the all order analysis of the second law of thermodynamics given in [2] to higher-form fluids, one can check that at arbitrarily high derivative orders, the second law forces the entropy current divergence in (3.8) to be a positive semi-definite quadratic form made out of various tensor structures in (4.12) and their derivatives. Since

${ }^{15}$ In the $q=1$ case, this condition reduces to

$$
\partial_{[\mu}\left(\frac{\mu}{T} v_{\nu]}^{1}\right)+\frac{u^{\lambda}}{T} F_{\lambda \mu \nu}=\frac{1}{T_{0}} \partial_{[\mu}\left(\sigma v_{\nu]}^{1}-\mathbf{k}^{\rho} A_{|\rho| \nu]}\right)=\frac{1}{T_{0}} \partial_{[\mu}\left(\zeta_{\nu]}^{1} \zeta_{1}^{\sigma} \mathbf{k}^{\rho} A_{\rho \sigma}-\mathbf{k}^{\rho} A_{|\rho| \nu]}\right)=0 .
$$

It is easy to see from here that this condition is generically non-trivial. To further supplement our intuition, let us choose a basis $\left(t, z, y^{a}\right)$ such that $\mathbf{k}^{\mu}=\delta^{\mu}{ }_{t}$ and $\ell_{1}^{\mu}=\delta^{\mu}{ }_{z}$. This renders the background metric and gauge field independent of the $t$ and $z$ coordinates. For simplicity, let us further choose $g_{\mu \nu}=\eta_{\mu \nu}$. The $[a b]$ components of the above equation then give a non-trivial condition

$$
\frac{1}{T_{0}} \partial_{[a} A_{b] t}=0
$$

which is clearly not satisfied for arbitrary $A_{\mu \nu}$. In the context of [18], this leads to the vanishing of their eq. (3.12) in equilibrium, which would otherwise contribute to entropy production.

${ }^{16}$ Even though ref. [24] did not impose (4.13) on their backgrounds, all their backgrounds do happen to satisfy (4.13). This means that all dipole charged black hole configurations studied in [24] are indeed equilibrium solutions. 
we have already ensured these to vanish on the solution (4.9), we are guaranteed to have a vanishing entropy current divergence at arbitrarily high orders in the derivative expansion.

Let us use this opportunity to point out a rather unconventional feature of hydrostatics in higher-form fluids as defined above. In 0-form hydrodynamics, one generally finds that requiring the existence of an equilibrium partition function does not give any new constraints on the constitutive relations, besides those already imposed by the second law of thermodynamics $[29,30]$. In other words, requiring the second law to hold on a set of constitutive relations is sufficient to guarantee the existence of an equilibrium partition function. In the higher-form case however, given that the existence of equilibrium relies on additional spacelike isometries, it is worth investigating if this still holds in full generality, in particular also when parity-invariance or charge conjugation invariance are not imposed. For concreteness, let us focus on the case of fluids with a 1-form symmetry and consider charge conjugation non-invariant derivative corrections to the constitutive relations of the form

$$
\begin{aligned}
\delta T^{\mu \nu} & =\frac{\mu}{T^{2}} v_{1}^{\rho} \partial_{\rho} T\left(T \frac{\partial \alpha_{1}}{\partial T} u^{\mu} u^{\nu}-\frac{\mu}{T} \frac{\partial \alpha_{1}}{\partial(\mu / T)} v_{1}^{\mu} v_{1}^{\nu}\right)-2 \alpha_{1} \frac{\mu}{T^{2}} v_{1}^{(\mu} \nabla^{\nu)} T-\nabla_{\mu}\left(\alpha_{1} \frac{\mu}{T} v_{1}^{\mu}\right) u^{\mu} u^{\nu}, \\
\delta J^{\mu \nu} & =\frac{2}{T} u^{[\mu}\left(\frac{\mu}{T^{2}} v_{1}^{\nu]} v_{1}^{\rho} \partial_{\rho} T \frac{\partial \alpha_{1}}{\partial \nu}+\alpha_{1} \frac{1}{T} \nabla^{\nu]} T\right) \\
\delta S^{\mu} & =2 \alpha_{1} \frac{\mu}{T^{3}} u^{[\rho} v_{1}^{\mu]} \partial_{\mu} T-\frac{1}{T} u_{\nu} \delta T^{\mu \nu}-\frac{\mu}{T} v_{\nu}^{1} \delta J^{\mu \nu},
\end{aligned}
$$

where $\alpha_{1}(T, \mu)$ is some independent transport coefficient. These expressions have been specifically engineered to satisfy the second law of thermodynamics with no entropy production. Interestingly, on the supposed solution (4.9) where $u^{\mu}$ and $v_{1}^{\mu}$ are aligned along (linear combinations of) isometries, most of the terms vanish, but the second to last term in the stress tensor in (4.14) and the last term in the charge current remain. This is clearly in tension with the equilibrium partition function because there are no first order parity-preserving (but charge conjugation non-invariant) scalars that can be written in equilibrium. Consequently, the partition function analysis sets $\alpha_{1}$ to zero. It appears, therefore, that for fluids with a higher-form symmetry, the equilibrium partition function analysis is imposing new constraints on the transport coefficients, which do not follow from an entropy current analysis. Although it is not a contradiction of any sort, it is in striking contrast with 0-form hydrodynamics where, by itself, the requirement of the second law to hold is sufficient to ensure a well-defined hydrostatic sector. ${ }^{17}$ We will return to these issues in a future publication [28].

\section{Surface dynamics of fluids with $q$-form symmetry}

In this section we study the surface transport properties of fluids carrying $q$-brane charge following $[10,12]$. We first introduce conservation equations for the surface dynamics and

\footnotetext{
${ }^{17}$ The analysis of [18] avoided these issues altogether by focusing on a sector which respects parity and charge-conjugation symmetry (which takes $v_{1}^{\mu} \rightarrow-v_{1}^{\mu}$ ). All the first order problematic terms of the kind (4.14) are not present if these requirements are imposed. However, it is not clear whether this continues to hold at higher orders in derivatives.
} 
then generalise the partition function discussed in the previous section in order to include the presence of a surface. We then perform a surface entropy current analysis and show that it agrees with the partition function expectations. Finally, we study capillary waves on the surface of the fluid and find signatures of anisotropy in the dispersion relation.

\subsection{Conservation equations and the second law of thermodynamics}

We introduce an interface/surface separating two different fluid phases by adding localised source contributions to the stress tensor and currents. Therefore, the full stress tensor, charge and entropy currents take the form

$$
\begin{aligned}
T^{\mu \nu} & =T_{\text {blk }}^{\mu \nu} \Theta(f)+T_{\text {sur }}^{\mu \nu} \widetilde{\delta}(f)+\ldots, \\
J & =J_{\text {blk }} \Theta(f)+J_{\text {sur }} \widetilde{\delta}(f)+\ldots, \\
S^{\mu} & =S_{\text {blk }}^{\mu} \Theta(f)+S_{\text {sur }}^{\mu} \widetilde{\delta}(f)+\ldots,
\end{aligned}
$$

where the dots represent higher order corrections in the thickness $\partial_{\rho} \delta(f)$ of the surface which we do not consider in the present paper. ${ }^{18}$ In (5.1) we have introduced the shapefield $f$ in terms of which the location of the surface is represented as $f=0$. The step function $\Theta(f)$ vanishes at $f=0$ while $\widetilde{\delta}(f)$ is the reparametrisation invariant delta function $\widetilde{\delta}(f)=\sqrt{-\gamma} / \sqrt{-g} \delta(f) . \gamma$ denotes the determinant of the induced metric on the surface, $\gamma_{\mu \nu}=g_{\mu \nu}-n_{\mu} n_{\nu}$, and $n_{\mu}=-\partial_{\mu} f /\left|\partial_{\mu} f \partial^{\mu} f\right|^{1 / 2}$ is the normal co-vector to the surface.

The conservation equations for the stress tensor were already considered in $[10,12]$ and the charge current conservation equation can be obtained by a simple generalisation of the results of $[36,37]$. These conservation laws take the form

$$
\widetilde{\nabla}_{\mu} T_{\mathrm{sur}}^{\mu \nu}-F_{\mathrm{sur}}^{\mu}=T_{\mathrm{blk}}^{\mu \nu} n_{\nu}, \quad \widetilde{\nabla}_{\mu} J_{\mathrm{sur}}^{\mu \mu_{1} \ldots \mu_{q}}=J_{\mathrm{bulk}}^{\mu \mu_{1} \ldots \mu_{q}} n_{\mu},
$$

with $F_{\text {sur }}^{\mu}=F^{\mu}{ }_{\mu_{1} \ldots \mu_{q+1}} J_{\text {sur }}^{\mu_{1} \ldots \mu_{q+1}} /(q+1)$ ! and in addition the system must obey the second law of thermodynamics

$$
\widetilde{\nabla}_{\mu} S_{\mathrm{sur}}^{\mu}-S_{\mathrm{blk}}^{\mu} n_{\mu} \geq 0
$$

together with the constraints $T_{\text {sur }}^{\mu \nu} n_{\mu}=J_{\text {sur }}^{\mu \mu_{1} \ldots \mu_{q}} n_{\mu}=S_{\text {sur }}^{\mu} n_{\mu}=0$. In eq. (5.2), we have introduced the surface projection of the background covariant derivative $\widetilde{\nabla}_{\mu} \equiv \gamma_{\mu}{ }^{\nu} \nabla_{\nu} \cdot{ }^{19}$ In the present paper, we choose a consistent truncation, as explained in $[10,12]$, in which the bulk currents are expanded up to first order in derivatives and the surface is kept at ideal order. This implies that we take the bulk stress tensor and currents to be those derived in the previous section at first order in derivatives, that is (2.1), (2.5), (2.7) together with (3.21). We will now consider equilibrium configurations and then perform a surface entropy current analysis.

\footnotetext{
${ }^{18}$ See [10] for a thorough analysis of these terms in the context of uncharged fluids.

${ }^{19}$ This covariant derivate should not be confused with the surface covariant derivative $\tilde{\nabla}_{\mu}$ introduced in [12]. The purpose of using the surface projection of the covariant derivative instead is to avoid having to work with the singular character of the delta function $\widetilde{\delta}(x)$.
} 


\subsection{Equilibrium partition function and entropy current analysis}

Analogously to the cases studied in [10, 12], one may write equilibrium partition functions for fluids with $q$-form symmetry in the presence of surfaces. Up to first order in derivatives, and under the assumptions of parity-invariance and charge conjugation invariance, the partition function takes the form ${ }^{20}$

$$
W=\frac{1}{T_{0}} \int_{\Sigma} d^{D-1} x \sqrt{-g} \mathcal{P}\left(\mathbf{k}^{2}, \sigma\right)+\frac{1}{T_{0}} \int_{\widetilde{\Sigma}} d^{D-2} \widetilde{x} \sqrt{-\gamma} \mathcal{C}\left(\mathbf{k}^{2}, \sigma\right)
$$

where $\Sigma$ is now a spatial hypersurface enclosed by the spatial codimension- 2 surface $\widetilde{\Sigma}$ at the boundary with coordinates $\widetilde{x}$. Since we aim to describe stationary configurations, we must have

$$
\mathcal{L}_{\mathbf{k}} f=0, \quad \mathcal{L}_{\ell_{i}} f=0 .
$$

By performing a variation with respect to the background metric and gauge fields one obtains the stress tensor and currents in the form (5.1), where, in particular, the surface stress tensor and charge current take the analogous form to (4.8) and with the exact same thermodynamic properties. We will explicitly derive these currents below using an entropy current analysis.

As shown in $[10,12]$, constraints on surface transport can be obtained by analysing the divergence of the surface entropy current. This analysis not only fixes the surface thermodynamics and currents, but also the value of $u^{\mu} n_{\mu}$ at leading order on the surface. As stated above, the surface currents must satisfy the constraints $T_{\text {sur }}^{\mu \nu} n_{\mu}=J_{\text {sur }}^{\mu \mu_{1} \ldots \mu_{q}} n_{\mu}=$ $S_{\mathrm{sur}}^{\mu} n_{\mu}=0$. This implies that at ideal order, and ignoring parity-odd effects, the surface currents take the form ${ }^{21}$

$$
\begin{aligned}
T_{\text {sur }}^{\mu \nu} & =(\mathcal{E}-\mathcal{Y}) \widetilde{u}^{\mu} \widetilde{u}^{\nu}-\mathcal{Y} \widetilde{\Gamma}^{\mu \nu}-(\mathcal{Y}+\mu \mathcal{Q}) \widetilde{\Pi}^{\mu \nu}, \\
J_{\text {sur }} & =\mathcal{Q} \widetilde{\operatorname{Vol}}_{(q+1)}, \\
S_{\text {sur }}^{\mu} & =\mathcal{S} \widetilde{u}^{\mu} .
\end{aligned}
$$

Here $\widetilde{u}^{\mu}=u^{\mu}-(u . n) n^{\mu}$ and $\widetilde{\Pi}^{\mu \nu}$ is a projector analogous to $\Pi^{\mu \nu}$ but constructed out the vectors $\widetilde{v}_{i}^{\mu}=v_{i}^{\mu}-\left(v_{i} . n\right) n^{\mu}$. Similarly, $\widetilde{\operatorname{Vol}}_{(q+1)}$ is the volume form introduced in (2.1) but with $u^{\mu}, v_{i}^{\mu}$ replaced by $\widetilde{u}^{\mu}, \widetilde{v}_{i}^{\mu}$. These two vectors satisfy $\widetilde{u} . n=\widetilde{v}_{i} \cdot n=0$. The projector $\widetilde{\Gamma}^{\mu \nu}$ is constructed using the induced metric so that $\widetilde{\Gamma}^{\mu \nu}=\gamma^{\mu \nu}+\widetilde{u}^{\mu} \widetilde{u}^{\nu}-\widetilde{\Pi}^{\mu \nu}$.

Noting that the bulk entropy current $S_{\text {blk }}^{\mu}$ is given by (2.7) and (3.21), requiring the second law (5.3) to be satisfied leads to

$$
\frac{\widetilde{u}^{\mu}}{T}\left(T \nabla_{\mu} \mathcal{S}+\mu \nabla_{\mu} \mathcal{Q}-\nabla_{\mu} \mathcal{E}\right)-\frac{1}{T}(\mathcal{E}-\mathcal{Y}-T \mathcal{S}-\mu \mathcal{Q}) \gamma^{\mu \nu} \widetilde{\nabla}_{\mu} \widetilde{u}_{\nu}-\frac{u . n}{T} \delta T_{\mathrm{blk}}^{\mu \nu} n_{\mu} n_{\nu} \geq 0,
$$

\footnotetext{
${ }^{20}$ Note that the condition (4.13) must be imposed on the background and a similar condition must be imposed on the surface in order for (5.4) to be an equilibrium partition function.

${ }^{21}$ It is possible to consider a component in the surface stress tensor of the form $\widetilde{u}^{(\mu} \widetilde{v}_{i}^{\mu)}$ which would ultimately be required to vanish by the second law of thermodynamics. For clarity of presentation, we have not considered it.
} 
where $\delta T_{\mathrm{blk}}^{\mu \nu}$ is the first order correction to the bulk stress tensor given in (3.21). In the present case, requiring positivity of the entropy current allows us to deduce the surface thermodynamics

$$
\mathrm{d} \mathcal{E}=T \mathrm{~d} \mathcal{S}+\mu \mathrm{d} \mathcal{Q}, \quad \mathcal{E}-\mathcal{Y}=T \mathcal{S}+\mu \mathcal{Q},
$$

as well as the expected condition $u . n=0$. This condition is expected to be modified at higher-orders [12]. In particular, do note that this analysis, similar to the case of the superfluid velocity in [12], does not fix the components $v_{i} . n$ at the surface. The stress tensor, current and thermodynamics obtained here agree with those that are readily derived from (5.4), once we identify $\mathcal{C}=-\mathcal{Y}$.

\subsection{Surface conservation equations}

Having derived the surface constitutive relations at ideal order, we can write down the first order equations of motion at the surface using (5.2). After imposing $u \cdot n=0$, the components of the $q$-form charge conservation law in (5.2) along with $\widetilde{u}^{\mu}$ and $\widetilde{\Pi}^{\mu \nu}$ are given by

$$
\begin{aligned}
u^{\mu} \nabla_{\mu} \mathcal{Q}+\mathcal{Q} \gamma^{\mu \nu} \widetilde{\nabla}_{\mu} u_{\nu}-\mathcal{Q} \widetilde{\Pi}^{\mu \nu} \nabla_{\mu} u_{\nu} & =0 \\
\widetilde{\Pi}_{\alpha}{ }^{\nu} \nabla_{\nu} \mathcal{Q}-\mathcal{Q} \widetilde{\Pi}_{\alpha \nu} u^{\mu} \nabla_{\mu} u^{\nu}+\mathcal{Q} \widetilde{\Pi}_{\alpha \nu} \widetilde{\nabla}_{\sigma} \widetilde{\Pi}^{\sigma \nu} & =Q \widetilde{v}_{\alpha}^{i}\left(v_{i}^{\mu} n_{\mu}\right),
\end{aligned}
$$

while those along $\widetilde{\Gamma}^{\mu \nu}$ are

$$
\widetilde{\Gamma}_{\alpha \mu}\left(u^{\nu} \nabla_{\nu} \widetilde{v}_{i}^{\mu}-\widetilde{v}_{i}^{\nu} \nabla_{\nu} u^{\mu}\right)=\widetilde{\Gamma}_{\alpha \mu}\left(\widetilde{v}_{i}^{\nu} \nabla_{\nu} \widetilde{v}_{j}^{\mu}-\widetilde{v}_{j}^{\nu} \nabla_{\nu} \widetilde{v}_{i}^{\mu}\right)=0 .
$$

These should be contrasted with the respective bulk $q$-form conservation laws in (2.13) and (2.15). The component of charge conservation along $n_{\mu}$ trivially vanishes. Upon using the charge conservation equations, the stress tensor conservation equation in (5.2) can be projected along $\widetilde{u}^{\mu}, \widetilde{\Pi}^{\mu \nu}$ and $\widetilde{\Gamma}^{\mu \nu}$ such that

$$
\begin{aligned}
T\left(u^{\mu} \nabla_{\mu} \mathcal{S}+\mathcal{S} \gamma^{\mu \nu} \widetilde{\nabla}_{\mu} u_{\nu}\right) & =0, \\
T \mathcal{S} \widetilde{\Pi}_{\alpha}{ }^{\nu}\left(\frac{1}{T} \nabla_{\nu} T+\mathfrak{a}_{\nu}\right) & =0, \\
(\mathcal{E}-\mathcal{Y}) \widetilde{\Gamma}_{\alpha}{ }^{\nu}\left(\frac{1}{T} \nabla_{\nu} T+\mathfrak{a}_{\nu}\right)+T \mathcal{Q} \widetilde{\Gamma}_{\alpha}{ }^{\nu}\left(\nabla_{\nu}\left(\frac{\mu}{T}\right)-\frac{\mu}{T} \gamma^{\mu}{ }_{\sigma} \nabla_{\mu} \widetilde{\Pi}^{\sigma}{ }_{\nu}\right) & =\widetilde{\Gamma}_{\alpha \nu} F_{\text {sur }}^{\nu},
\end{aligned}
$$

which are analogous to the bulk equations given in (2.17). Finally, projecting the stress tensor conservation equation along $n_{\mu}$ we get the Young-Laplace equation

$$
-(\mathcal{E}-\mathcal{Y}) u^{\mu} u^{\nu} \nabla_{\mu} n_{\nu}+\mathcal{Y} \nabla_{\mu} n^{\mu}+\mu \mathcal{Q} \widetilde{\Pi}^{\mu \nu} \nabla_{\mu} n_{\nu}=P-\mu Q \Pi^{\mu \nu} n_{\mu} n_{\nu},
$$

which provides an equation of motion for the shape-field. We will solve these equations at the linear level in the next subsection.

\subsection{Surface waves}

In this subsection we study the nature of linearised fluctuations about an equilibrium configuration. For simplicity, we work on a flat background with metric $\eta_{\mu \nu}$ and vanishing 
gauge field. We pick a basis $x^{\mu}=\left\{t, z^{i}, y^{a}, r\right\}$ and work around the equilibrium solution given $b^{22}$

$$
u^{\mu}=\delta_{t}{ }^{\mu}, \quad v_{i}{ }^{\mu}=\delta_{i}{ }^{\mu}, \quad T=T_{0}, \quad \mu=\mu_{0}, \quad f=r .
$$

It follows that $n_{\mu}=-\delta^{r}{ }_{\mu}$. This solution obviously satisfies the bulk equations of motion. To solve the surface equations of motion, we must further require $P\left(T_{0}\right)=0$. Performing a small perturbation around this solution and using that $u^{\mu} n_{\mu}=0$ implies

$$
\delta u^{t}=0, \quad \delta v_{i}^{t}=\delta u_{i}, \quad \delta v_{i}^{j}=-\delta v_{i}^{j}, \quad \delta u^{r}=-\partial_{t} \delta f .
$$

Furthermore, the vanishing Lie derivative conditions (5.10) imply

$$
\begin{array}{ll}
\mathrm{L}_{1} \equiv \partial_{t} \delta v_{i}^{a}-\partial_{i} \delta u^{a}=0, & \partial_{i} \delta v_{j}{ }^{a}-\partial_{j} \delta v_{i}{ }^{a}=0, \\
\mathrm{~L}_{2} \equiv \partial_{t} \delta v_{i}^{r}-\partial_{i} \delta u^{r}=\partial_{t}\left(\delta v_{i}^{r}+\partial_{i} \delta f\right)=0, & \partial_{i} \delta v_{j}^{r}-\partial_{j} \delta v_{i}^{r}=0,
\end{array}
$$

which can be seen as determining $\delta v_{i}{ }^{a}$ and $\delta v_{i}{ }^{r}$ respectively. Note that the antisymmetric modes in $\delta v_{i}{ }^{j}$ are not physical due to the underlying $\mathrm{SO}(q)$ symmetry. Therefore, the remaining degrees of freedom we need to solve for are $\delta T, \delta \mu, \delta u^{i}, \delta u^{a}$ and $\delta f$. Let us first look at the boundary equations of motion. The Young-Laplace condition provides an equation of motion for $\delta f$ that takes the form

$$
\mathrm{YL} \equiv \mathcal{E} \partial_{t}^{2} \delta f-(\mathcal{Y}+\mu \mathcal{Q}) \delta^{i j} \partial_{i} \partial_{j} \delta f-\mathcal{Y} \delta^{a b} \partial_{a} \partial_{b} \delta f-S \delta T-Q \delta \mu .
$$

The surface conservation equations (5.11) imply

$$
\begin{aligned}
& \mathrm{S}_{1} \equiv \partial_{t} \delta \mathcal{S}+\mathcal{S} \partial_{i} \delta u^{i}+\mathcal{S} \partial_{a} \delta u^{a}=0, \\
& \mathrm{~S}_{2} \equiv \frac{1}{T} \partial_{i} \delta T+\partial_{t} \delta u_{i}=0, \\
& \mathrm{~S}_{3} \equiv(\mathcal{E}-\mathcal{Y})\left(\frac{1}{T} \partial_{a} \delta T+\partial_{t} \delta u_{a}\right)+T \mathcal{Q}\left(\partial_{a} \delta\left(\frac{\mu}{T}\right)-\frac{\mu}{T} \partial_{i} \delta v^{i}{ }_{a}\right)=0, \\
& \mathrm{~S}_{4} \equiv \partial_{t} \delta \mathcal{Q}+\mathcal{Q} \partial_{a} \delta u^{a}=0, \\
& \mathrm{~S}_{5} \equiv \partial_{i} \delta \mathcal{Q}+\mathcal{Q} \partial_{a} \delta v_{i}{ }^{a}+Q\left(\delta v_{i}^{r}+\partial_{i} \delta f\right)=0 .
\end{aligned}
$$

Finally, the bulk equations of motion (2.13), (2.15) and (2.16) at the linear level are given by

$$
\begin{aligned}
\mathrm{B}_{1} & \equiv \partial_{t} \delta S+S \partial_{i} \delta u^{i}+S \partial_{a} \delta u^{a}-S \partial_{t} \partial_{r} \delta f=0, \\
\mathrm{~B}_{2} & \equiv \frac{1}{T} \partial_{i} \delta T+\partial_{t} \delta u_{i}=0, \\
\mathrm{~B}_{3} & \equiv E\left(\frac{1}{T} \partial_{a} \delta T+\partial_{t} \delta u_{a}\right)+T Q\left(\partial_{a} \delta\left(\frac{\mu}{T}\right)-\frac{\mu}{T} \partial_{i} \delta v^{i}{ }_{a}\right)=0, \\
\mathrm{~B}_{3}^{\prime} & \equiv E\left(\frac{1}{T} \partial_{r} \delta T-\partial_{t}^{2} \delta f\right)+T Q\left(\partial_{r} \delta\left(\frac{\mu}{T}\right)-\frac{\mu}{T} \partial_{i} \delta v^{i}{ }_{r}\right)=0, \\
\mathrm{~B}_{4} & \equiv \partial_{t} \delta Q+Q \partial_{a} \delta u^{a}-Q \partial_{t} \partial_{r} \delta f=0, \\
\mathrm{~B}_{5} & \equiv \partial_{i} \delta Q+Q \partial_{a} \delta v_{i}{ }^{a}+Q \partial_{r} \delta v_{i}^{r}=0 .
\end{aligned}
$$

\footnotetext{
${ }^{22}$ For simplicity, we have assumed that $f$ has no dependence on the $z^{i}$ coordinates.
} 
We focus on plane wave solutions to these equations which behave as $\mathrm{e}^{i\left(\omega t-k_{i} z^{i}-\ell_{a} y^{a}\right)} \mathrm{e}^{-\kappa r}$, where $\omega$ is the frequency of the wave, $k_{i}$ is the wavenumber along the $(p-q)$ directions, $\ell_{a}$ the wavenumber along the $q$ anisotropic directions and $\kappa$ is a damping factor. Equations $\mathrm{L}_{1}, \mathrm{~L}_{2}, \mathrm{~B}_{2}, \mathrm{~S}_{2}$ are then immediately solved by choosing

$$
\delta v_{i}^{a}=-\frac{k_{i}}{\omega} \delta u^{a}, \quad \delta v_{i}^{r}=i k_{i} \delta f, \quad \delta u^{i}=\frac{k^{i}}{\omega} \frac{1}{T} \delta T .
$$

This also makes $\mathrm{B}_{5}$ and $\mathrm{S}_{5}$ linearly dependent on $\mathrm{B}_{4}$ and $\mathrm{S}_{4}$ respectively. The $\delta u^{a}$ components of the velocity are obtained by solving $\mathrm{B}_{3}$ and $\mathrm{S}_{3}$ such that

$$
\delta u^{a}=\frac{\omega \ell^{a}}{\omega^{2} E-k^{2} Q \mu}(S \delta T+Q \delta \mu),\left.\quad \delta u^{a}\right|_{r \rightarrow 0}=\frac{\omega \ell^{a}}{\omega^{2}(\mathcal{E}-\mathcal{Y})-k^{2} \mathcal{Q} \mu}(\mathcal{S} \delta T+\mathcal{Q} \delta \mu) .
$$

Finally, we can turn to the scalar degrees of freedom $\delta T$ and $\delta \mu$ which are given by solutions of $\mathrm{B}_{1}, \mathrm{~B}_{3}^{\prime}, \mathrm{B}_{4}$ in the bulk and $\mathrm{S}_{1}, \mathrm{~S}_{4}$ and $\mathrm{YL}$ at the surface. At the surface we have

$$
\begin{aligned}
& \left(\frac{k^{2}}{\omega^{2}} \frac{\mathcal{S}}{T}+\frac{\ell^{2} \mathcal{S}^{2}}{\omega^{2}(\mathcal{E}-\mathcal{Y})-k^{2} \mathcal{Q} \mu}-\mathcal{X}_{T T}\right) \delta T+\left(\frac{\ell^{2} \mathcal{Q S}}{\omega^{2}(\mathcal{E}-\mathcal{Y})-k^{2} \mathcal{Q} \mu}-\mathcal{X}_{T \mu}\right) \delta \mu=0, \\
& \left(\frac{\ell^{2} \mathcal{Q S}}{\omega^{2}(\mathcal{E}-\mathcal{Y})-k^{2} \mathcal{Q} \mu}-\mathcal{X}_{T \mu}\right) \delta T+\left(\frac{\ell^{2} \mathcal{Q}^{2}}{\omega^{2}(\mathcal{E}-\mathcal{Y})-k^{2} \mathcal{Q} \mu}-\mathcal{X}_{\mu \mu}\right) \delta \mu=0, \\
& \left(-\omega^{2} \mathcal{E}+k^{2} \mu \mathcal{Q}+\left(k^{2}+\ell^{2}\right) \mathcal{Y}\right) \delta f=S \delta T+Q \delta \mu
\end{aligned}
$$

which provide boundary conditions for the bulk equations of motion

$$
\begin{aligned}
& \left(\frac{k^{2}}{\omega^{2}} \frac{S}{T}+\frac{\left(\ell^{2}-\kappa^{2}\right) S^{2}}{\omega^{2} E-k^{2} Q \mu}-\chi_{T T}\right) \delta T+\left(\frac{\left(\ell^{2}-\kappa^{2}\right) S Q}{\omega^{2} E-k^{2} Q \mu}-\chi_{T \mu}\right) \delta \mu=0, \\
& \left(\frac{\left(\ell^{2}-\kappa^{2}\right) Q S}{\omega^{2} E-k^{2} Q \mu}-\chi_{T \mu}\right) \delta T+\left(\frac{\left(\ell^{2}-\kappa^{2}\right) Q^{2}}{\omega^{2} E-k^{2} Q \mu}-\chi_{\mu \mu}\right) \delta \mu=0, \\
& \delta f=\frac{\kappa}{\omega^{2} E-Q \mu k^{2}}(S \delta T+Q \delta \mu) .
\end{aligned}
$$

Here we have defined $k^{2}=k_{i} k^{i}, \ell^{2}=\ell_{a} \ell^{a}$ as well as the susceptibility matrices

$$
\begin{aligned}
& \mathcal{X}_{T T}=-\frac{\partial^{2} \mathcal{Y}}{\partial T^{2}}=\frac{\partial \mathcal{S}}{\partial T}, \quad \mathcal{X}_{T \mu}=-\frac{\partial^{2} \mathcal{Y}}{\partial T \partial \mu}=\frac{\partial \mathcal{S}}{\partial \mu}=\frac{\partial \mathcal{Q}}{\partial T}, \quad \mathcal{X}_{\mu \mu}=-\frac{\partial^{2} \mathcal{Y}}{\partial \mu^{2}}=\frac{\partial \mathcal{Q}}{\partial \mu}, \\
& \chi_{T T}=\frac{\partial^{2} P}{\partial T^{2}}=\frac{\partial S}{\partial T}, \quad \chi_{T \mu}=\frac{\partial^{2} P}{\partial T \partial \mu}=\frac{\partial S}{\partial \mu}=\frac{\partial Q}{\partial T}, \quad \chi_{\mu \mu}=\frac{\partial^{2} P}{\partial \mu^{2}}=\frac{\partial Q}{\partial \mu} .
\end{aligned}
$$

From eqs. (5.33) and (5.36) we can read out the frequency $\omega$, which is given by

$$
\omega= \pm \sqrt{\frac{Q \mu k^{2}+\kappa k^{2} \mu \mathcal{Q}+\kappa\left(k^{2}+\ell^{2}\right) \mathcal{Y}}{E+\kappa \mathcal{E}}}
$$

and is required for the consistency of the solution to $\delta f$. Finally, we have consistency conditions involving $\delta T$ and $\delta \mu$, which will determine $\kappa, k^{2}$ and $\ell^{2}$, namely

$$
\left|\begin{array}{cc}
\frac{k^{2}}{\omega^{2}} \frac{S}{T}+\frac{\left(\ell^{2}-\kappa^{2}\right) S^{2}}{\omega^{2} E-k^{2} Q \mu}-\chi_{T T} & \frac{\left(\ell^{2}-\kappa^{2}\right) S Q}{\omega^{2} E-k^{2} Q \mu}-\chi_{T \mu} \\
\frac{\left(\ell^{2}-\kappa^{2}\right) S Q}{\omega^{2} E-k^{2} Q \mu}-\chi_{T \mu} & \frac{\left(\ell^{2}-\kappa^{2}\right) Q^{2}}{\omega^{2} E-k^{2} Q \mu}-\chi_{\mu \mu}
\end{array}\right|=0,
$$


and the following vanishing determinants at the surface

$$
\begin{aligned}
& \left|\begin{array}{cc}
\frac{k^{2}}{\omega^{2}} \frac{\mathcal{S}}{T}+\frac{\ell^{2} \mathcal{S}^{2}}{\omega^{2}(\mathcal{E}-\mathcal{Y})-k^{2} \mathcal{Q} \mu}-\mathcal{X}_{T T} & \frac{\ell^{2} \mathcal{Q S}}{\omega^{2}(\mathcal{E}-\mathcal{Y})-k^{2} \mathcal{Q} \mu}-\mathcal{X}_{T \mu} \\
\frac{\ell^{2} \mathcal{Q S}}{\omega^{2}(\mathcal{E}-\mathcal{Y})-k^{2} \mathcal{Q} \mu}-\mathcal{X}_{T \mu} & \frac{\ell^{2} \mathcal{Q}^{2}}{\omega^{2}(\mathcal{E}-\mathcal{Y})-k^{2} \mathcal{Q} \mu}-\mathcal{X}_{\mu \mu}
\end{array}\right|=0, \\
& \left|\begin{array}{cc}
\frac{k^{2}}{\omega^{2}} \frac{S}{T}+\frac{\left(\ell^{2}-\kappa^{2}\right) S^{2}}{\omega^{2} E-k^{2} Q \mu}-\chi_{T T} & \frac{\left(\ell^{2}-\kappa^{2}\right) S Q}{\omega^{2} E-k^{2} Q \mu}-\chi_{T \mu} \\
\frac{k^{2}}{\omega^{2}} \frac{\mathcal{S}}{T}+\frac{\ell^{2} \mathcal{S}^{2}}{\omega^{2}(\mathcal{E}-\mathcal{Y})-k^{2} \mathcal{Q} \mu}-\mathcal{X}_{T T} & \frac{\ell^{2} \mathcal{Q} \mathcal{S}}{\omega^{2}(\mathcal{E}-\mathcal{Y})-k^{2} \mathcal{Q} \mu}-\mathcal{X}_{T \mu}
\end{array}\right|=0 .
\end{aligned}
$$

Eqs. (5.39) to (5.42) completely characterise the wave fluctuations of the $q$-form fluid with a surface.

We will now study the dispersion relation of capillary waves in a particular approximation scheme. For simplicity, we focus on the simplest case in which $\mathcal{Y}$ is a constant function of the temperature and chemical potential. This implies that $\mathcal{S}=\mathcal{Q}=\mathcal{X}_{T T}=$ $\mathcal{X}_{T \mu}=\mathcal{X}_{\mu \mu}=0$, hence eqs. (5.41) and (5.42) are automatically satisfied. In order to solve (5.40) we focus on long-wavelength perturbations so that $k_{i} \sim \tau$ and $\ell_{a} \sim \tau$ for a small parameter $\tau$ and $k \neq 0$. In addition we consider the regime of small charge $Q$ so that $Q, \chi_{T \mu}, \chi_{\mu \mu} \sim \epsilon$ for a small parameter $\epsilon$. In this situation (5.40) leads to

$$
\kappa=|k|-\frac{\left(k^{2}+\ell^{2}\right)}{2 Q S^{2}} \mathcal{Y}\left(\chi_{T T} Q-\chi_{T \mu} S\right)+\mathcal{O}\left(\tau^{4}, \epsilon\right), \quad \omega= \pm \sqrt{\frac{\kappa\left(k^{2}+\ell^{2}\right) \mathcal{Y}}{T S+\mathcal{E} \kappa}}+\mathcal{O}(\epsilon),
$$

which in turn yields the dispersion relation

$$
\omega=\sqrt{\frac{k\left(k^{2}+\ell^{2}\right) \mathcal{Y}}{S T+\mathcal{E} k}}\left(1-\left(k^{2}+\ell^{2}\right) \mathcal{Y} T \frac{\left(\chi_{T T} Q-\chi_{T \mu} S\right)}{4 k Q S(\mathcal{E} k+S T)}+\mathcal{O}\left(k^{7 / 2}, \epsilon\right)\right) .
$$

It is instructive to consider this expression in a particular limit. An interesting situation is the case in which the perturbation only occurs along the $(p-q)$ transverse directions so that $\ell=0 .{ }^{23}$ In this context, the dispersion relation takes the form

$$
\omega=k^{3 / 2} \sqrt{\frac{\mathcal{Y}}{S T}}\left(1-k \mathcal{Y} \frac{\left(\chi_{T T} Q-\chi_{T \mu} S\right)}{4 Q S^{2}}+\mathcal{O}\left(k^{7 / 2}, \epsilon\right)\right),
$$

where we have assumed that $k$ is small enough such that $\mathcal{E} k \ll S T$. The leading $k^{3 / 2}$ behaviour is the classical result for the dispersion relation for capillary waves of an uncharged fluid (see e.g. [12]), while the sub-leading term of order $k^{5 / 2}$ is a small deviation due to the presence of the dipole charge density $Q$.

Another interesting situation is one in which clear signatures of anisotropy are observed. Consider now the case in which there is no perturbation along the $(p-q)$ directions so that $k_{i}=0$. In the same regime where $\ell_{a} \sim \tau$ and $Q, \chi_{T \mu}, \chi_{\mu \mu} \sim \epsilon$, eq. (5.40) now leads to

$$
\omega^{2}=\frac{\ell^{2} \mathcal{Y} \kappa}{T S+\mathcal{E} \kappa}+\mathcal{O}(\epsilon), \quad \kappa=-\ell^{2} \frac{\mathcal{Y}}{Q S^{2}}\left(\chi_{T T} Q-\chi_{T \mu} S\right)+\mathcal{O}\left(\tau^{4}, \epsilon\right)
$$

\footnotetext{
${ }^{23}$ The same scaling behaviour is observed if we take the perturbations to be the same in both directors, i.e. when $k^{2}=\ell^{2}$.
} 
so that the dispersion relation takes the form

$$
\omega= \pm \ell^{2} \sqrt{\frac{\mathcal{Y}^{2}}{T S^{2}} \frac{\mathrm{d} \log (Q / S)}{\mathrm{d} T}}+\mathcal{O}\left(\tau^{4}, \epsilon\right)
$$

This behaviour is a radical departure from the usual dispersion relation of capillary waves for isotropic fluids which behaves as (5.45) and a clear signature of the presence of microscopic anisotropies. In order not to have unstable modes on the surface one must have that $\mathrm{d} \log (Q / S) / d T>0$. If this condition does not hold, (5.47) suggests that the surface would not form in any physical situation as a small perturbation along the $\Pi_{\mu \nu}$ directions would exponentially grow in time. In such cases, it would be interesting to understand the nature of the resulting surface instability. However, do note that this potential instability could be cured by considering the more physically relevant situation in which $\mathcal{Y}$ depends non-trivially on $T$ and $\mu$. In fact, in such context it would be interesting to study sound modes on the surface, analogously to [12]. We leave this possibility for future work.

\section{Discussion}

In this paper we have introduced a framework for building effective theories of hydrodynamics with higher-form symmetries. In particular, we have developed in detail the case of fluids with a single $q$-form symmetry to first order in derivatives. After defining the ideal order dynamics in section 2 , we have found in section 3 that the dissipative and parity-even sector of the theory up to first order in derivatives is characterised by $q+7$ independent transport coefficients for $q>1$ and 7 for $q=1$, once Onsager's relation is imposed. In comparison with the $q=1$ case of $[18,19]$, there is one extra transport coefficient for $q>1$ corresponding to the shear viscosity in the $\mathrm{SO}(q)$ sector and $q-1$ extra current resistivities. In section 5 we have generalised these results in order to include the presence of an interface separating distinct fluid phases and studied capillary waves on the interface, which show signatures of anisotropy. This analysis turns out to be similar to the analysis carried out in the case of superfluids in [12].

The work presented here suggests a few possible extensions:

The hydrostatic sector. In section 4 we have constructed the most general equilibrium partition function under the assumption of one timelike and $q$ spacelike isometries. We observed that this partition function is more general than the one presented in [18] for $q=1$ and that it generalises for $q>1$ the solution provided by the free energy obtained in [24] for $q=1$. Ref. [18] assumed that the isometries, in addition to having a vanishing Lie bracket, had a vanishing inner product. This, however, is not necessarily the case as we have explained.

Insisting on the existence of a hydrostatic sector for fluids with $q$-form symmetry, regardless of the microscopic origin of the vectors $v_{i}$, we noticed that equilibrium configurations do not exist unless constraints are imposed on the background, in particular, the existence of $q$ spacelike commuting isometries and an additional constraint, namely, $\mathrm{d}\left(\sigma \operatorname{Vol}_{q}-\imath_{\mathbf{k}} A_{q+1}\right)=0$. This condition guarantees that the equilibrium configurations obtained from (4.7) do not produce entropy. We understand that this is a peculiar feature 
of fluids with higher-form symmetries as usually equilibrium only requires the existence of a timelike isometry and no spatial isometries or extra conditions. The need for these features originate from the fact that in equilibrium one must satisfy the charge conservation equation

$$
\nabla_{\mu}\left(Q T v_{1}^{\mu}\right)=0
$$

where we have specialised to the $q=1$ case for simplicity. A simple way to satisfy this relation is to assume $v_{1}^{\mu}$ to be a linear combination of background Killing vector fields at the expense of having to introduce the ad-hoc requirement that $\mathrm{d}\left(\sigma \operatorname{Vol}_{q}-\imath_{\mathbf{k}} A_{q+1}\right)=0$. On top of this, as we commented in section 4.2, the equilibrium partition function seems to impose new constraints on transport coefficients that do not follow from the second law of thermodynamics. These restrictions are clearly unsatisfactory and we intend to return to this issue in a forthcoming publication [28].

Gravitational duals. As mentioned in the introduction, one of the main motivations of this work was to understand the structure of long-wavelength perturbations of black branes in supergravity. In the context of the fluid/gravity correspondence [38] and, more generally, in the context of the blackfold approach [39, 40], one may test theories of hydrodynamics by appropriately perturbing certain classes of black brane geometries. It is therefore interesting to consider gravitational duals to these theories and perturb them in a derivative expansion. In the case $q=1$ and in Anti-de Sitter space this was considered in [20]. In a future publication [35], we consider a more general class of black brane geometries valid for all $p, q, Q$ and show that, for a constant external gauge field, their perturbations are characterised by the existence of 8 independent transport coefficients for $q>1$ in addition to the conservation equations for the stress tensor and current appearing as constraint equations. In particular, we show that this particular class of geometries satisfy the relations (3.28) away from the isotropic limit. Since the second relation in (3.28) is also satisfied for the class considered in [20], this suggests that the relations (3.28) may be universal for generic theories of gravity, at least those theories without higher-derivative corrections.

Fluids with multiple higher-form currents. The case of fluids with multiple higherform currents is of particular interest in the context of supergravity and string theory as generic black brane bound states in string theory may carry multiple higher-form charges. In particular, in the case of the D3-F1 bound state in type IIB string theory, the effective fluid carries two 2-form currents $j_{2}$ and $J_{2}$ dual to the NSNS two-form $B_{2}$ and the 2 -form RR field, respectively, in addition to a 4 -form current $J_{4}$ dual to the 4-form RR field [27]. However, the current conservation equations are not trivial, instead they must satisfy

$$
\nabla_{\mu} j_{2}^{\mu \nu}=0, \quad \nabla_{\mu} J_{4}^{\mu \mu_{1} \mu_{2} \mu_{3}}=0, \quad \nabla_{\mu} J_{2}^{\mu \nu}=\frac{1}{3 !} J_{4}^{\mu_{1} \mu_{2} \mu_{3} \nu} H_{3 \mu_{1} \mu_{2} \mu_{3}},
$$

where $H_{3 \mu_{1} \mu_{2} \mu_{3}}=\mathrm{d} B_{2 \mu_{1} \mu_{2} \mu_{3}}$. It would be interesting to address these systems in the future and to consider the most general type of hydrodynamic theory that can arise from supergravity [27]. 
Another context where fluids with multiple higher-form currents have a role to play is in the context of the effective theory of charge density waves and states with dynamical defects [21, 41-43]. The framework introduced in this work is capable of dealing with these cases and to provide a systematic construction of effective hydrodynamic theories with multiple higher-form symmetries.

\section{Acknowledgments}

We would like to thank J. Bhattacharya and D. Hofman for various helpful discussions. JA would like to thank R. Emparan for a motivational discussion on this subject. AJ would like to thank Perimeter Institute, where part of this project was done, for hospitality. JA is partly supported by the Netherlands Organization for Scientific Research (NWO). AJ is supported by the Durham Doctoral Scholarship offered by Durham University. The work of AVP has been supported by The Danish Council for Independent Research - Natural Sciences (FNU), DFF-4002-00307. AVP gratefully acknowledges support from UC Berkeley where some of the research for this paper was carried out.

\section{A Currents in another fluid frame}

In this appendix we briefly compare the transport coefficients in the frame chosen in section 3.1.1 with those presented in [18] for $q=1$. The two frames differ from each other due to the transport coefficient $\Theta$ in the current (3.21). Using the frame transformation (3.5) in order to remove this term from the current we require

$$
\bar{\delta} v_{1}^{\mu}=2 \Theta \Gamma^{\mu \nu} v_{1}^{\lambda} \nabla_{(\nu} u_{\lambda)},
$$

which due to (3.5) adds an extra term to the stress tensor with one index along $v_{1}^{\mu}$ and another along the normal directions $\bar{\delta} v_{1}^{\mu}$. Comparing with eqs. (3.9)-(3.14) of [18] and using (3.21) we identify the transport coefficients

$$
\eta_{\|}=Q \mu \Theta, \quad \eta_{\perp}=\eta_{\Gamma}, \quad r_{\perp}=\mathfrak{D} T, \quad r_{\|}=\mu \Omega_{1}, \quad \zeta_{\|}=\zeta_{\Pi}, \quad \zeta_{\perp}=\zeta_{\Gamma}, \quad \zeta_{\times}=\zeta,
$$

where the coefficients $\eta_{\|}, \eta_{\perp}, r_{\perp}, r_{\|}, \zeta_{\|}, \zeta_{\perp}$ and $\zeta_{\times}$were introduced in [18].

Open Access. This article is distributed under the terms of the Creative Commons Attribution License (CC-BY 4.0), which permits any use, distribution and reproduction in any medium, provided the original author(s) and source are credited.

\section{References}

[1] F.M. Haehl, R. Loganayagam and M. Rangamani, The eightfold way to dissipation, Phys. Rev. Lett. 114 (2015) 201601 [arXiv:1412.1090] [INSPIRE].

[2] F.M. Haehl, R. Loganayagam and M. Rangamani, Adiabatic hydrodynamics: The eightfold way to dissipation, JHEP 05 (2015) 060 [arXiv: 1502.00636] [INSPIRE].

[3] A. Jain, Theory of non-Abelian superfluid dynamics, Phys. Rev. D 95 (2017) 121701 [arXiv: 1610.05797] [INSPIRE]. 
[4] F.M. Haehl, R. Loganayagam and M. Rangamani, Topological $\sigma$-models $\mathcal{E}$ dissipative hydrodynamics, JHEP 04 (2016) 039 [arXiv: 1511.07809] [INSPIRE].

[5] M. Crossley, P. Glorioso and H. Liu, Effective field theory of dissipative fluids, JHEP 09 (2017) 095 [arXiv:1511.03646] [INSPIRE].

[6] P. Glorioso, M. Crossley and H. Liu, Effective field theory of dissipative fluids (II): classical limit, dynamical KMS symmetry and entropy current, JHEP 09 (2017) 096 [arXiv: 1701.07817] [INSPIRE].

[7] P. Glorioso, H. Liu and S. Rajagopal, Global Anomalies, Discrete Symmetries and Hydrodynamic Effective Actions, arXiv:1710.03768 [INSPIRE].

[8] K. Jensen, N. Pinzani-Fokeeva and A. Yarom, Dissipative hydrodynamics in superspace, arXiv: 1701.07436 [INSPIRE].

[9] F.M. Haehl, R. Loganayagam and M. Rangamani, Two roads to hydrodynamic effective actions: a comparison, arXiv:1701.07896 [INSPIRE].

[10] J. Armas, J. Bhattacharya and N. Kundu, Surface transport in plasma-balls, JHEP 06 (2016) 015 [arXiv:1512.08514] [INSPIRE].

[11] P. Kovtun, Thermodynamics of polarized relativistic matter, JHEP 07 (2016) 028 [arXiv: 1606.01226] [INSPIRE].

[12] J. Armas, J. Bhattacharya, A. Jain and N. Kundu, On the surface of superfluids, JHEP 06 (2017) 090 [arXiv:1612.08088] [INSPIRE].

[13] K. Jensen, Aspects of hot Galilean field theory, JHEP 04 (2015) 123 [arXiv:1411.7024] [INSPIRE].

[14] N. Banerjee, S. Dutta and A. Jain, Null Fluids - A New Viewpoint of Galilean Fluids, Phys. Rev. D 93 (2016) 105020 [arXiv:1509.04718] [InSPIRE].

[15] A. Jain, Galilean Anomalies and Their Effect on Hydrodynamics, Phys. Rev. D 93 (2016) 065007 [arXiv: 1509.05777] [INSPIRE].

[16] N. Banerjee, S. Dutta and A. Jain, First Order Galilean Superfluid Dynamics, Phys. Rev. D 96 (2017) 065004 [arXiv:1612.01550] [INSPIRE].

[17] D. Schubring, Dissipative String Fluids, Phys. Rev. D 91 (2015) 043518 [arXiv:1412.3135] [INSPIRE].

[18] S. Grozdanov, D.M. Hofman and N. Iqbal, Generalized global symmetries and dissipative magnetohydrodynamics, Phys. Rev. D 95 (2017) 096003 [arXiv: 1610.07392] [INSPIRE].

[19] J. Hernandez and P. Kovtun, Relativistic magnetohydrodynamics, JHEP 05 (2017) 001 [arXiv: 1703.08757] [INSPIRE].

[20] S. Grozdanov and N. Poovuttikul, Generalised global symmetries and magnetohydrodynamic waves in a strongly interacting holographic plasma, arXiv:1707.04182 [INSPIRE].

[21] S. Grozdanov and N. Poovuttikul, Generalized global symmetries in states with dynamical defects: The case of the transverse sound in field theory and holography, Phys. Rev. D 97 (2018) 106005 [arXiv:1801.03199] [INSPIRE].

[22] D. Gaiotto, A. Kapustin, N. Seiberg and B. Willett, Generalized Global Symmetries, JHEP 02 (2015) 172 [arXiv:1412.5148] [INSPIRE].

[23] R. Emparan, T. Harmark, V. Niarchos and N.A. Obers, Blackfolds in Supergravity and String Theory, JHEP 08 (2011) 154 [arXiv:1106.4428] [INSPIRE]. 
[24] M.M. Caldarelli, R. Emparan and B. Van Pol, Higher-dimensional Rotating Charged Black Holes, JHEP 04 (2011) 013 [arXiv: 1012.4517] [InSPIRE].

[25] R. Emparan, V.E. Hubeny and M. Rangamani, Effective hydrodynamics of black D3-branes, JHEP 06 (2013) 035 [arXiv: 1303.3563] [INSPIRE].

[26] A. Di Dato, J. Gath and A.V. Pedersen, Probing the Hydrodynamic Limit of (Super)gravity, JHEP 04 (2015) 171 [arXiv: 1501.05441] [INSPIRE].

[27] J. Armas, J. Gath, V. Niarchos, N.A. Obers and A.V. Pedersen, Forced Fluid Dynamics from Blackfolds in General Supergravity Backgrounds, JHEP 10 (2016) 154 [arXiv:1606.09644] [INSPIRE].

[28] J. Armas and A. Jain, to appear.

[29] S. Bhattacharyya, Entropy current and equilibrium partition function in fluid dynamics, JHEP 08 (2014) 165 [arXiv: 1312.0220] [INSPIRE].

[30] S. Bhattacharyya, Entropy Current from Partition Function: One Example, JHEP 07 (2014) 139 [arXiv: 1403.7639] [INSPIRE].

[31] L. Landau and E. Lifshitz, Fluid Mechanics, Course of Theoretical Physics, Vol. 6, Elsevier (1987).

[32] J. Gath and A.V. Pedersen, Viscous asymptotically flat Reissner-Nordström black branes, JHEP 03 (2014) 059 [arXiv: 1302.5480] [INSPIRE].

[33] D.T. Son and A.O. Starinets, Viscosity, Black Holes and Quantum Field Theory, Ann. Rev. Nucl. Part. Sci. 57 (2007) 95 [arXiv:0704.0240] [InSPIRE].

[34] P. Kovtun, Lectures on hydrodynamic fluctuations in relativistic theories, J. Phys. A 45 (2012) 473001 [arXiv:1205.5040] [InSPIRE].

[35] J. Armas, J. Gath and A.V. Pedersen, to appear.

[36] J. Armas, J. Gath and N.A. Obers, Black Branes as Piezoelectrics, Phys. Rev. Lett. 109 (2012) 241101 [arXiv:1209.2127] [INSPIRE].

[37] J. Armas, J. Gath and N.A. Obers, Electroelasticity of Charged Black Branes, JHEP 10 (2013) 035 [arXiv: 1307.0504] [INSPIRE].

[38] S. Bhattacharyya, V.E. Hubeny, S. Minwalla and M. Rangamani, Nonlinear Fluid Dynamics from Gravity, JHEP 02 (2008) 045 [arXiv:0712.2456] [INSPIRE].

[39] R. Emparan, T. Harmark, V. Niarchos and N.A. Obers, World-Volume Effective Theory for Higher-Dimensional Black Holes, Phys. Rev. Lett. 102 (2009) 191301 [arXiv:0902.0427] [INSPIRE].

[40] R. Emparan, T. Harmark, V. Niarchos and N.A. Obers, Essentials of Blackfold Dynamics, JHEP 03 (2010) 063 [arXiv:0910.1601] [INSPIRE].

[41] T. Andrade, M. Baggioli, A. Krikun and N. Poovuttikul, Pinning of longitudinal phonons in holographic spontaneous helices, JHEP 02 (2018) 085 [arXiv: 1708.08306] [INSPIRE].

[42] L. Alberte, M. Ammon, M. Baggioli, A. Jiménez-Alba and O. Pujolàs, Holographic Phonons, Phys. Rev. Lett. 120 (2018) 171602 [arXiv:1711.03100] [INSPIRE].

[43] A. Amoretti, D. Areán, B. Goutéraux and D. Musso, Effective holographic theory of charge density waves, Phys. Rev. D 97 (2018) 086017 [arXiv:1711.06610] [INSPIRE]. 